\title{
The Use of Microwave Energy in Dental Prosthesis
}

\author{
Célia M. Rizzatti-Barbosa, Altair A. Del Bel Cury \\ and Renata C. M. Rodrigues Garcia \\ State University of Campinas (Unicamp) \\ Brazil
}

\section{Introduction}

Acrylic resins are used as denture base materials since 1937 (Craig, 1993) and heat-cured Poly(methyl-methacrylate) (PMMA) (Lay et al., 2004) composed by methyl methacrylate monomers chains is the most popular of them. Virtually, dentures are constructed from conventional polymer/monomer (Lay et al., 2004) processed by heat water-bath system and compression molding technique (Ganzarolli et al., 2002). It is prepared by mixing the monomer and polymer in a specific ratio, resulting in a dough mass that is packed and pressed in a flask for polymerization (Ganzarolli et al., 2007). Addition polymerization of PMMA requires the activation of the initiator (benzoyl peroxide) to provide free radicals. Polymerization takes place as the free radicals open the double bonds of the methyl methacrylate, creating a chain reaction where the monomer attaches to polymer free radicals (Bartoloni et al., 2000). When the polymerization reaction is activated by heat, the monomers form polymeric chains joined by high energy linkings (crossed-links) and this reaction would finish when all monomers have supposal been reacted (Machado et al., 2004).

Although water-bath polymerization is extensively used to process PMMA, new resins and processing methods have been proposed in order to obtain better physical properties and to simplify the technique (Souza Jr. et al., 2006). Heating curing, chemical curing by pouring technique, light curing and microwave curing resin have been extensively studied for denture base processing (Harman, 1949; Nishii, 1968; Jerolimov et al., 1989; Urabe et al., 1999).

Polymerization of heat-cured PMMA is usually carried out in a temperature-controlled water bath for at least 9 hours. However, the use of microwave energy to polymerize PMMA decreases the time to three minutes only, producing acrylic resin bases with the same quality as those polymerized by water bath technique (Nishii, 1968; Kimura et al, 1983; Hayden, 1986; Jerolimov et al., 1989; Del Bel Cury et al., 1994; Rizzatti-Barbosa et al., 1995; Braun et al., 1988; Ganzarolli et al., 2002; Yunus et al., 2005; Rizzatti-Barbosa et al., 2009). The microwave polymerization technique has advantages such us less equipment is required (Schnieder et al., 2002), the method is fast and clean, the final product has the same quality of physical properties, and it presents better accuracy of fit, resulting in improvement adaptation of denture base (Rizzatti-Barbosa et al., 1995; Rodrigues-Garcia et al., 1996; Compagnoni et al., 2004; Shibayama et al., 2009). The advantages of microwave heating over conventional heating are: (1) the inside and outside of substance are almost equally heated, 
and (2) temperature rises rapidly (Lai et al., 2004). Microwave energy acts on the monomer promoting an uniform and immediate heating of the polymer mass (Machado et al., 2004), that activates the decomposition of benzoyl peroxide, the reaction initiator, and quickly yields free radicals for the polymerization process (Sarac et al., 2005), which decreases in the same proportion as polymerization increases (Compagnoni et al., 2004).

Polymerization of PMMA by microwave energy is possible because methyl methacrylate (MMA) is a polar liquid at room temperature, and microwaves create an electronmagnetic field inside microwave oven that allows the MMA molecules to orient themselves at a frequency of $2450 \mathrm{MHz}$ (Sanders et al., 1991). Numerous polarized molecules are flipped over rapidly and generate heat due to molecular friction. Because of this, microwave heating is independent of the thermal conductivity; therefore, curing cycles involving the application of rapid heat may be used without the development of a high exothermic temperature.

The use of microwave energy was first reported in 1968 by Nishii as an alternative PMMA processing method and has become increasingly popular as to conventional water-bath processing (Schnieder et al., 2002). Several studies showed that physical properties of denture base resins polymerized with microwave energy or by heat water bath (conventional method) exhibited similar behavior being both of methods adequate to be used in denture acrylic resins fabrication (Kimura et al., 1983; Kimura et al., 1984; Kimura et al., 1987). Some studies reported that it is possible to polymerize acrylic resin in a very short time using microwave energy, however the metal flask must be substituted by the fiberreinforced plastic (FRP) (Kimura et al., 1983, 1984 and 1985; Reitz et al., 1985; RizzattiBarbosa et al., 1995).

Since that, several studies have been investigated different properties of acrylic resin when processed by microwave energy. In Brazil, the first microwave research was reported in 1994 by Del Bel Cury et al., who investigated physical properties of acrylic resin processed by microwave energy compared to water bath. Their results showed differences among the resins that were attributed to the composition of acrylic resins.

\section{Solubility, residual monomer and water sorption}

The solubility is a property of acrylic resin, representing the not reacted substances releasing (residual monomer, plasticizers and initializers). It is characterized as an undesirable property of resins, since they should be insoluble in oral fluids. Residues releasing from a polymerized resin base can promote tissular reactions in users of prosthesis (Machado et al., 2009). From this point of view, the degree of conversion is one of the most important characteristic of resin, on account of the high residual monomer levels that could be unreacted. Its presence has an adverse effect on physical and mechanical properties (Yunus et al., 1994) as well as on the biocompatibility (Ilbay et al., 1994). Acrylic resin also presents water sorption that is directly related to the polar properties of resin molecules, the physical process of water diffusion through intermolecular space (Takahashi et al., 1998), and the amount of residual monomer in the polymerized mass (Wong et al., 1999). Thus, polymerization degree is directly related to resin's ability of absorbing water (Meloto et al., 2006). Polymerization takes place as the free radicals open the double bonds of the methyl-methacrylate, creating a chain reaction where the monomer attaches to polymer free radicals. The degree of monomer conversion to polymer structure of PMMA is a measure of the carbon double bonds $(\mathrm{C}=\mathrm{C})$ converted into carbon single bonds (C-C) (Bartoloni et al., 2000). 
According to Azzarri et al. (2003), from the appropriate selection of power and the resin's curing time, it is possible to optimise the level of residual monomer and a low cytotoxicity keeping at the same time better mechanical properties. Because of the quick and instantaneous heating, the polymerization of monomers could be damaged by the microwave energy. However, classical studies have been shown that microwavepolymerized acrylic resin presents lower or the same residual monomer levels relative to conventionally polymerized resins (McCabe \& Basker, 1976; Austin \& Basker, 1980; Austin \& Basker, 1982; Huggett et al., 1984; DeClerck, 1987; Truong \& Thomasz, 1988; Al Doori et al., 1988; Sadamori et al., 1994; lbay et al., 1994; Yunus et al., 1994; Shlosberg et al., 1989; Jacob et al., 1997). Other researches showed resin polymerized by microwave energy presented similar solubily, residual monomer or water sorption levels than conventional or fast polymerization process.

\begin{tabular}{|c|c|c|c|}
\hline $\begin{array}{l}\text { Authors and } \\
\text { date }\end{array}$ & Materials and groups & $\begin{array}{l}\text { Methods and } \\
\text { measurements }\end{array}$ & $\begin{array}{l}\text { Comments and } \\
\text { conclusions }\end{array}$ \\
\hline $\begin{array}{l}\text { Urban et al. } \\
\text { (2007) }\end{array}$ & $\begin{array}{l}\text { Four hard chair-side } \\
\text { reline resins (Duraliner } \\
\text { II-D, Kooliner-K, Tokuso } \\
\text { Rebase Fast-TRF and Ufi } \\
\text { Gel hard-UGH) (short } \\
\text { and long polymerization } \\
\text { cycles ) } \\
\text { One heat-polymerized } \\
\text { denture base resin } \\
\text { (Lucitone 550-L) (short } \\
\text { and long polymerization } \\
\text { cycles ) } \\
\text { Immersion in hot water }\end{array}$ & $\begin{array}{l}\text { High performance liquid } \\
\text { chromatography, } \\
\text { expressed as a } \\
\text { percentage of residual } \\
\text { monomer. }\end{array}$ & $\begin{array}{l}\text { Statistical differences } \\
\text { in residual monomer } \\
\text { percentage were found } \\
\text { among all materials (K } \\
\text { >D > UGH > LLong } \\
>\mathrm{TRF}>\text { LShort). } \\
\text { The reduction in } \\
\text { residual monomer } \\
\text { promoted by water } \\
\text { bath and microwave } \\
\text { post-polymerization } \\
\text { treatments could } \\
\text { improve the } \\
\text { mechanical properties } \\
\text { and biocompatibility of } \\
\text { the relining and } \\
\text { denture base materials. }\end{array}$ \\
\hline $\begin{array}{l}\text { Meloto et al. } \\
\text { (2006) }\end{array}$ & $\begin{array}{l}\text { Vipi Cril heat- } \\
\text { polymerized acrylic resin } \\
\left(73^{\circ} \mathrm{C} \text { for } 9 \mathrm{~h}\right) \text { and Vipi } \\
\text { Wave microwave resin } \\
(20 \mathrm{~min} / 90 \mathrm{~W} \text { and } 5 \\
\mathrm{min} / 450 \mathrm{~W})\end{array}$ & $\begin{array}{l}\text { Specimens }(\mathrm{m} 1) \text { were } \\
\text { stored in distilled water } \\
\text { at } 37 \pm 1^{\circ} \mathrm{C} \text { during } 30 \\
\text { days }(\mathrm{m} 2) \text {, and weighed. } \\
\text { Water sorption }(\mathrm{g} / \mathrm{cm} 3) \\
\text { was calculated using the } \\
\text { formula: WS }=(\mathrm{m} 2- \\
\mathrm{m} 1) / \mathrm{V} \text {. }\end{array}$ & $\begin{array}{l}\text { No difference was } \\
\text { found between the } \\
\text { group fabricated using } \\
\text { water bath and } \\
\text { microwave } \\
\text { polymerization. }\end{array}$ \\
\hline $\begin{array}{l}\text { Machado et al. } \\
\text { (2004) }\end{array}$ & $\begin{array}{l}\text { Vipi Cril heat-cured } \\
\text { acrylic resin (water bath - } \\
74 \pm 1^{\circ} \mathrm{C} \text { for } 9 \mathrm{~h} \text {; } \\
\text { Microwave oven }-500 \\
\text { W/ } 3 \text { min.) }\end{array}$ & $\begin{array}{l}\text { Solubility test and } \\
\text { percentile solubility }\end{array}$ & $\begin{array}{l}\text { The association } \\
\text { between } \\
\text { polymerization by } \\
\text { microwave irradiation } \\
\text { and mechanical } \\
\text { polishing showed } \\
\text { lower percentile } \\
\text { solubility, indicating } \\
\text { lower residual } \\
\text { substances releasing. }\end{array}$ \\
\hline
\end{tabular}




\begin{tabular}{|c|c|c|c|}
\hline $\begin{array}{c}\text { Authors and } \\
\text { date }\end{array}$ & Materials and groups & $\begin{array}{l}\text { Methods and } \\
\text { measurements }\end{array}$ & $\begin{array}{l}\text { Comments and } \\
\text { conclusions }\end{array}$ \\
\hline Lai et al. (2004) & $\begin{array}{l}\text { PMMA denture base } \\
\text { polymer (Optilon-399) - } \\
\text { (water-bath at } 708 \text { C for } 9 \\
\text { h; resin blocks was } \\
\text { processed at } 80,160,240 \text {, } \\
\text { and } 560 \mathrm{~W} \text { for } 15,10,7 \text {, } \\
\text { and } 2 \text { min, separately + } \\
\text { additional } 2 \text { min at } 560 \\
\text { W. }\end{array}$ & $\begin{array}{l}\text { Internal temperature - } \\
\text { Thermocouples. } \\
\text { Solubility - samples } \\
\text { weigh percentage of after } \\
\text { specific treatment. }\end{array}$ & $\begin{array}{l}\text { Large difference in the } \\
\text { curing temperature } \\
\text { was observed. } \\
\text { The results of the } \\
\text { temperature } \\
\text { measurement and the } \\
\text { solubility analysis } \\
\text { indicated that a } \\
\text { microwave oven for } \\
\text { curing resin was much } \\
\text { faster than a } \\
\text { conventional water- } \\
\text { bath, and the degree of } \\
\text { curing also increased a } \\
\text { little. } \\
\text { Microwave energy can } \\
\text { efficiently polymerize } \\
\text { and cure denture base } \\
\text { polymer from the } \\
\text { results of insoluble } \\
\text { weight percent and the } \\
\text { curing temperature. }\end{array}$ \\
\hline $\begin{array}{l}\text { Phoenix et al. } \\
\text { (2004) }\end{array}$ & $\begin{array}{l}6 \text { commonly used } \\
\text { polymethyl methacrylate } \\
\text { denture base resins. } \\
\text { ADA Specification No. } \\
12 . \\
\text { Thermal assessments } \\
\text { (differential scanning } \\
\text { calorimetry and dynamic } \\
\text { mechanical analysis). }\end{array}$ & Sorption and solubility & $\begin{array}{l}\text { The microwaveable } \\
\text { resins displayed better } \\
\text { results than other } \\
\text { denture base resins } \\
\text { included in the } \\
\text { investigation. }\end{array}$ \\
\hline $\begin{array}{l}\text { Azzarri et al. } \\
\text { (2003) }\end{array}$ & $\begin{array}{l}\text { Four different conditions } \\
\text { of power and curing } \\
\text { time }\end{array}$ & $\begin{array}{l}\text { Release of residual } \\
\text { monomer in water was } \\
\text { evaluated by } \\
\text { spectrophotometric } \\
\text { method up to } 24 \mathrm{~h} \text {. }\end{array}$ & $\begin{array}{l}\text { It is possible to } \\
\text { optimise the level of } \\
\text { residual monomer and } \\
\text { a low cytotoxicity } \\
\text { keeping from the } \\
\text { appropriate selection } \\
\text { of power and time of } \\
\text { curing of the resin. }\end{array}$ \\
\hline $\begin{array}{l}\text { Oliveira et al. } \\
\text { (2003) }\end{array}$ & $\begin{array}{l}\text { Acron MC }-500 \mathrm{~W} / 3 \mathrm{~m} \\
\text { or } 4.5 \mathrm{~m} \text {. } \\
\text { One simple flask } \\
\text { centrally placed on the } \\
\text { turning plate; two flasks, } \\
\text { one in the centre and the } \\
\text { other peripherally placed } \\
\text { in the plate; two flasks } \\
\text { centrally, one above and } \\
\text { the other below. }\end{array}$ & $\begin{array}{l}\text { Monomer levels - } \\
\text { spectrophotometry at } \\
206 \mathrm{~nm} \text {. }\end{array}$ & $\begin{array}{l}\text { Monomer release was } \\
\text { affected by the position } \\
\text { of the flask. }\end{array}$ \\
\hline
\end{tabular}




\begin{tabular}{|c|c|c|c|}
\hline $\begin{array}{c}\text { Authors and } \\
\text { date }\end{array}$ & Materials and groups & $\begin{array}{l}\text { Methods and } \\
\text { measurements }\end{array}$ & $\begin{array}{l}\text { Comments and } \\
\text { conclusions }\end{array}$ \\
\hline $\begin{array}{l}\text { Bartoloni et al. } \\
(2000)\end{array}$ & $\begin{array}{l}\text { Lucitone } 199 \AA \\
\text { (conventional long-cure), } \\
\text { Ac-celar 20® (rapid } \\
\text { cure), and Acron MC }( \\
\text { (microwave cure) }\end{array}$ & $\begin{array}{l}\text { Fourier transform } \\
\text { infrared spectrometry } \\
\text { (FTIR) }\end{array}$ & $\begin{array}{l}\text { All curing methods } \\
\text { obtained similar } \\
\text { degrees of conversion } \\
\text { determined by FTIR. }\end{array}$ \\
\hline $\begin{array}{l}\text { Blagojevic et al. } \\
\text { (1999) }\end{array}$ & $\begin{array}{l}\text { TS1195 ; Acron MC; } \\
\text { Biocryl NR. } \\
\text { Microwave oven for } 3 \\
\text { min at } 600 \mathrm{~W} \text { and hot- } \\
\text { water bath for } 14 \mathrm{~h} \text { at } 70 \\
{ }^{\circ} \mathrm{C} \text { followed by a } 3 \mathrm{~h} \\
\text { terminal boil. }\end{array}$ & $\begin{array}{l}\text { Gas liquid } \\
\text { chromatography. }\end{array}$ & $\begin{array}{l}\text { General trend } \\
\text { suggested that the } \\
\text { water-bath method } \\
\text { enhanced the degree of } \\
\text { polymerization } \\
\text { resulting in a lower } \\
\text { level of residual } \\
\text { monomer. Residual } \\
\text { monomer was } \\
\text { considerably lower for } \\
\text { TS1195, Acron MC and } \\
\text { Biocryl NR following } \\
\text { water both } \\
\text { polymerization. }\end{array}$ \\
\hline $\begin{array}{l}\text { Del Bel Cury et } \\
\text { al. (1994) }\end{array}$ & $\begin{array}{l}\text { Acrylic resins: Acron MC } \\
\text { (microwave - } \\
500 \mathrm{~W} / 3 \mathrm{~m} \text { ); Lucitone } 550 \\
\text { (water bath - } \\
73 \mathrm{oC} / 90 \mathrm{~m} \text { ); Ortho-Class } \\
\text { (self polymerized). }\end{array}$ & $\begin{array}{l}\text { Water sorption and } \\
\text { solubility - load of } \\
\text { samples }\end{array}$ & $\begin{array}{l}\text { There were differences } \\
\text { among tested materials } \\
\text { that can be resulted } \\
\text { from composition and } \\
\text { polymerization } \\
\text { methods. }\end{array}$ \\
\hline
\end{tabular}

Table 1. Studies on solubility, residual monomer and water sorption of microwaved resin

\section{Porosity and color stability}

Porosity has been attributed to a variety of factors such as air entrapped during mixing, monomer contraction during polymerization, monomer vaporization associated with the exothermic reaction, and the presence of residual monomer (Keller \& Lautenschlager, 1985; Wolfaardt et al., 1986). According to Tager (Tager, 1978) porosity is a property of solids that relates to their structure and is expressed in the presence of voids (pores) between separate grains, layers, crystals, and other elements of a coarse structure of a solid. This definition emphasizes the fact that the concept of porosity can be applied to solids, and that pores are spaces not between molecules, but between super molecule structures. Other authors (Taylor, 1941; Sweeney et al., 1942;) demonstrated that insufficient mixing of monomer and polymer, processing temperatures higher than $74^{\circ} \mathrm{C}$, packing of the mold, and inadequate compression on the flask may cause porosity in denture base resin. Depending on polymerization conditions, up to $11 \%$ porosity has been observed associated with decreased mechanical properties and poor esthetics (Keller \& Lautenschlager, 1985) and the potential harboring of organisms and retention of fluids (Davenport, 1970; Compagnoni et al., 2004). Irregularities and porosities present on denture surfaces offer a favorable niche to retain stain and bacterial biofilm, and because of this denture base polymers are susceptible to color shifting. It depends not only of the polymerization method but also on the chemical characteristics of the material (Polyzois et al., 1999). Considering the processing methods for 
acrylic resins, some authors (Austin et al., 1982; May et al.,1992) have stated that polymerization for short period of time promotes higher color instability.

Porosity and its consequences have been studied to resin processed by microwave energy (Reitz et al., 1985; Wolfaardt et al., 1986; De Clerck, 1987; Al Doori et al., 1988; Truong \& Thomasz, 1988; Levin et al., 1989; Shlosberg et al., 1989; Alkhatib et al., 1990; Bafile et al.,1991; Sadamori et al., 1994; Ilbay et al.,1994; ) and some authors verified that it depends on the base thickness (Sanders et al., 1987) or the selection of the material, despite of the microwave processing. (Yannikakis et al., 2002). Prevalent studies on porosity and its consequences are listed on Table 2 and show similar results when comparing microwave and water bath or light cure processing.

\begin{tabular}{|c|c|c|c|}
\hline $\begin{array}{c}\text { Authors and } \\
\text { date }\end{array}$ & Materials and groups & $\begin{array}{l}\text { Methods and } \\
\text { measurements }\end{array}$ & $\begin{array}{l}\text { Comments and } \\
\text { conclusions }\end{array}$ \\
\hline $\begin{array}{l}\text { Rizzatti- } \\
\text { Barbosa et al. } \\
(2009)\end{array}$ & $\begin{array}{l}\text { Water bath-cured resin } \\
\text { (Vipi-Cryl) }-(74 \circ \mathrm{C} \text { for } \\
9 \text { hours) } \\
\text { Microwave-cured resin } \\
\text { (Vipi-Wave) }-(90 \mathrm{~W} \text { for } \\
13 \text { minutes }+450 \mathrm{~W} \text { for } \\
5 \text { minutes) }\end{array}$ & $\begin{array}{l}\text { Solution of permanent } \\
\text { black ink for } 12 \text { hours. } \\
\text { Number of pores per } \\
\text { area. }\end{array}$ & $\begin{array}{l}\text { Microwave curing is not a } \\
\text { factor that alters surface } \\
\text { superficial porosity. }\end{array}$ \\
\hline $\begin{array}{l}\text { Assunção et al. } \\
\text { (2009) }\end{array}$ & $\begin{array}{l}\text { Heat cured resin } \\
\text { (Microwave } \\
\text { polymerization - } 500 \mathrm{~W} \\
\text { for } 3 \text { minutes; water } \\
\text { bath }-74^{\circ} \mathrm{C} \text { for } 9 \text { hours) }\end{array}$ & $\begin{array}{l}\text { Spectrophotometer that } \\
\text { measured visible } \\
\text { ultraviolet reflection. } \\
\text { Color changes } \\
\text { calculatedby the } \\
\text { Commission } \\
\text { Internationale de } \\
\text { l'Eclairage system - D65 } \\
\text { standard illumination. }\end{array}$ & $\begin{array}{l}\text { The color of denture teeth } \\
\text { was not affected by the } \\
\text { polymerization methods, } \\
\text { as there was no } \\
\text { significant difference } \\
\text { between microwave and } \\
\text { conventional } \\
\text { polymerization. }\end{array}$ \\
\hline $\begin{array}{l}\text { Paranhos et al. } \\
\text { (2009) }\end{array}$ & $\begin{array}{l}\text { Microwave- } \\
\text { polymerized acrylic } \\
\text { resin (after immersion } \\
\text { in } 0.5 \% \mathrm{NaOCl}, 1 \% \\
\mathrm{NaOCl} \text {, and } \\
\text { Clorox/Calgon) }\end{array}$ & $\begin{array}{l}\text { Portable colorimeter } \\
\text { (Color-guide } 45 / 0) \text {. } \\
\text { Color changes }(\Delta \mathrm{E}) \text { were } \\
\text { calculated with the use of } \\
\text { CIELab color space }\end{array}$ & $\begin{array}{l}\text { No statistically significant } \\
\text { differences ( } p>0.05) \\
\text { among the solutions for } \\
\text { color teeth stability. }\end{array}$ \\
\hline $\begin{array}{l}\text { Pero et al. } \\
\text { (2007) }\end{array}$ & $\begin{array}{l}\text { Onda-Cryl (microwave: } \\
500 \mathrm{~W} \text { for } 3 \mathrm{~min} \text {; } 90 \mathrm{~W} \\
\text { for } 13 \mathrm{~min}+500 \mathrm{~W} \text { for } \\
90 \mathrm{~s} ; 320 \mathrm{~W} \text { for } 3 \mathrm{~min}+ \\
0 \mathrm{~W} \text { for } 4 \mathrm{~min}+720 \mathrm{~W} \\
\text { for } 3 \mathrm{~min} \text { ); Classico } \\
\text { (water bath: } 74 \circ \mathrm{C} \text { for } \\
\text { 9h). } \\
\text { Sample thickness: } 2.0 \\
\mathrm{~mm} ; 3.5 \mathrm{~mm} ; 5.0 \mathrm{~mm} \text {. }\end{array}$ & $\begin{array}{l}\text { Polymers and water } \\
\text { (classical sorption } \\
\text { method and mercury } \\
\text { porosimetry - porosity of } \\
\text { a sorbet is estimated } \\
\text { quantitatively by total } \\
\text { pore volume (W0)). }\end{array}$ & $\begin{array}{l}\text { The influence of } \\
\text { microwave } \\
\text { polymerization cycle on } \\
\text { porosity of acrylic resin } \\
\text { appears only on the } \\
\text { thinnest specimens } \\
(2.0 \mathrm{~mm}) \text {. } \\
\text { Water bath } \\
\text { polymerization group } \\
\text { presented similar } \\
\text { porosity results for } \\
\text { specimens of } \\
\text { all tested } \\
\text { thicknesses. }\end{array}$ \\
\hline
\end{tabular}




\begin{tabular}{|c|c|c|c|}
\hline $\begin{array}{c}\text { Authors and } \\
\text { date }\end{array}$ & Materials and groups & $\begin{array}{c}\text { Methods and } \\
\text { measurements }\end{array}$ & $\begin{array}{c}\text { Comments and } \\
\text { conclusions }\end{array}$ \\
\hline Lai et al. (2004) & $\begin{array}{l}\text { PMMA denture base } \\
\text { polymer (Optilon- } 399) \\
\text { - (water-bath at } 70^{\circ} \mathrm{C} \\
\text { for } 9 \text { h; resin blocks was } \\
\text { processed at } 80 \text {, } \\
160,240 \text {, and } 560 \mathrm{~W} \text { for } \\
15,10,7 \text {, and } 2 \text { min, } \\
\text { separately + additional } \\
2 \text { min at } 560 \mathrm{~W} \text {. } \\
\text { Thickness samples was } \\
\text { less than } 10 \text { mm. }\end{array}$ & $\begin{array}{l}\text { Pulse echo C-scans - } \\
\text { Testech ultrasonic tester } \\
\text { (model VI-100) - } \\
\text { frequency of } 10 \mathrm{MHz} / \\
25.4 \mathrm{~mm}\end{array}$ & $\begin{array}{l}\text { The amount of porosity } \\
\text { increased with an } \\
\text { increase in microwave } \\
\text { energy level. } \\
\text { It is important to control } \\
\text { temperature accurately } \\
\text { and ensure correct timing } \\
\text { to minimize porosity } \\
\text { when microwave } \\
\text { polymerization is used. }\end{array}$ \\
\hline $\begin{array}{l}\text { Compagnoni et } \\
\text { al. (2004) }\end{array}$ & $\begin{array}{l}\text { Acrylic resin samples: } \\
\text { Onda-Cryl, microwave } \\
\text { polymerized ( } 500 \mathrm{~W} \text { for } \\
3 \text { minutes, } 90 \mathrm{~W} \text { for } 13 \\
\text { minutes }+500 \mathrm{~W} \text { for } 90 \\
\text { seconds, } 320 \mathrm{~W} \text { for } 3 \\
\text { minutes }+0 \mathrm{~W} \text { for } 4 \\
\text { minutes }+720 \mathrm{~W} \text { for } 3 \\
\text { minutes); Clássico, } \\
\text { heat-polymerized } \\
\text { (water } 74 \text { o C } / 9 \mathrm{~h} \text { ). }\end{array}$ & $\begin{array}{l}\text { Porosity - measurement } \\
\text { of the specimen volume } \\
\text { before and after its } \\
\text { immersion in water. } \\
\text { Specimen was weighed } \\
\text { in air and in water to } \\
\text { calculate percent mean } \\
\text { porosity. The absolute } \\
\text { density of acrylic resin } \\
\text { was used to calculate the } \\
\text { percent mean porosity. }\end{array}$ & $\begin{array}{l}\text { No differences in mean } \\
\text { porosity were found } \\
\text { among resin specimens } \\
\text { polymerized by } \\
\text { microwave energy. } \\
\text { The porosity of } \\
\text { microwave-polymerized } \\
\text { resin was similar in } \\
\text { porosity to the heat- } \\
\text { polymerized resin. }\end{array}$ \\
\hline $\begin{array}{l}\text { Oliveira et al. } \\
(2003)\end{array}$ & $\begin{array}{l}\text { Acron } \mathrm{MC}-500 \mathrm{~W} / 3 \mathrm{~m} \\
\text { or } 4.5 \mathrm{~m} \text {. } \\
\text { One simple flask } \\
\text { centrally placed on the } \\
\text { turning plate; two } \\
\text { flasks, one in the centre } \\
\text { and the other } \\
\text { peripherally placed in } \\
\text { the plate; two flasks } \\
\text { centrally, one above } \\
\text { and the other below. }\end{array}$ & $\begin{array}{l}\text { Porosity - permanent ink } \\
\text { and counting the porous } \\
\text { in a stereo light } \\
\text { microscope. }\end{array}$ & $\begin{array}{l}\text { There is no difference } \\
\text { among the groups. }\end{array}$ \\
\hline $\begin{array}{l}\text { Yannikakis et } \\
\text { al. (2002) }\end{array}$ & $\begin{array}{l}\text { Two heat-activated } \\
\text { denture base resins } \\
\text { (short and long } \\
\text { microwave cycle) } \\
\text { One conventional } \\
\text { (Paladon 65) (water } \\
\text { bath cured) } \\
\text { One designed for } \\
\text { microwave } \\
\text { polymerization (Acron } \\
\text { MC) } \\
3 \text { mm and } 6 \text { mm } \\
\text { thickness. }\end{array}$ & $\begin{array}{l}\text { Photographed under a } \\
\text { microscope at } \times 100 \\
\text { magnification. } \\
\text { Pore were measured with } \\
\text { a digital planimeter. } \\
\text { Total area of pores per } \\
\text { surface was calculated in } \\
\text { percentage form }\end{array}$ & $\begin{array}{l}\text { There are no pores in the } \\
\text { group polymerized in } \\
\text { water bath. } \\
\text { Minor porosity was } \\
\text { identified in thin areas } \\
\text { and more severe porosity } \\
\text { in thicker areas of } \\
\text { conventional resin } \\
\text { specimens that } \\
\text { underwent microwave } \\
\text { polymerization. } \\
\text { No significant porosity } \\
\text { was observed in the resin } \\
\text { designed specifically for } \\
\text { microwave } \\
\text { polymerization. } \\
\text { Severe porosity in thicker }\end{array}$ \\
\hline
\end{tabular}




\begin{tabular}{|c|c|c|c|}
\hline $\begin{array}{l}\text { Authors and } \\
\text { date }\end{array}$ & Materials and groups & $\begin{array}{l}\text { Methods and } \\
\text { measurements }\end{array}$ & $\begin{array}{l}\text { Comments and } \\
\text { conclusions }\end{array}$ \\
\hline & & & $\begin{array}{l}\text { areas of conventional } \\
\text { resin specimens that } \\
\text { underwent microwave } \\
\text { polymerization, and no } \\
\text { significant porosity in the } \\
\text { resin designed } \\
\text { specifically for this } \\
\text { technique. }\end{array}$ \\
\hline $\begin{array}{l}\text { Rodrigues } \\
\text { Garcia et al. } \\
\text { (1996) }\end{array}$ & $\begin{array}{l}\text { Denture bases of } \\
\text { conventional acrylic } \\
\text { resin (water bath } 73 \mathrm{oC} / \\
9 \text { hours) and specific } \\
\text { resin for microwave } \\
\text { polymerization ( } 500 \\
\text { W/3m). } \\
\text { Dentures were relined } \\
\text { by addition method. }\end{array}$ & $\begin{array}{l}\text { Porosity (immersion of } \\
\text { samples in a solution } \\
\text { black - pores were } \\
\text { counted / stereo light } \\
\text { microscope under } \\
\text { magnification of } 6.3 \mathrm{x} \text { ) }\end{array}$ & $\begin{array}{l}\text { Conventional resin cured } \\
\text { by water bath or } \\
\text { microwaves energy } \\
\text { showed the highest } \\
\text { number of pores after } \\
\text { relining }\end{array}$ \\
\hline $\begin{array}{l}\text { May et al. } \\
\text { (1996) }\end{array}$ & $\begin{array}{l}\text { Seven conventional } \\
\text { denture base materials } \\
\text { One microwave } \\
\text { heat cured denture } \\
\text { base material processed } \\
\text { with the microwave } \\
\text { method. } \\
\text { Conditions of } \\
\text { accelerated aging to test } \\
\text { for color stability. }\end{array}$ & $\begin{array}{l}\text { Color measurements were } \\
\text { made before weathering } \\
\text { and at } 300,600 \text {, and } 900 \\
\text { hours. }\end{array}$ & $\begin{array}{l}\text { Color changes occurred } \\
\text { after accelerated aging in } \\
\text { heat-cured denture base } \\
\text { resins and Acron GC } \\
\text { microwave acrylic resins } \\
\text { processed with the } \\
\text { microwave. }\end{array}$ \\
\hline
\end{tabular}

Table 2. Studies on porosity and color stability of microwaved resin

\section{Hardness, transverse strength, flexural strength, shear bond strength, tensile bond strength, impact strength, roughness, and modulus of elasticity}

Curing processes have been modified in order to improve the physical and mechanical properties of those materials, and also to afford the technical work of the professionals. The relationship between the physicochemical characteristics and the final properties of a material are of fundamental importance to obtain a resin with the desired properties. The constituent polymer of the powder exhibits a high average molecular weight and a broad of molecular weight distribution. The powder - liquid ratio determines the time dependence of monomer conversion and the rate of polymerization for the formation of the cross-linked network that grows throughout the chains of the base polymer (Wallace et al., 1994). Both characteristics of the polymerization rate and conversion, are proportional to the concentration of the reactive species as well as to the instantaneous temperature (Urabe et al., 1999).

For the microwave-cured acrylic resins, it has been demonstrated that the temperature developed during the reaction is not constant: it increases quickly at the beginning, goes through a maximum and then decays, being able to reach peaks of the order of $150-200{ }^{\circ} \mathrm{C}$, depending on the working conditions (Gourdinne et al., 1979; Jacob et al., 1997). Hence, both the power of the microwave and the time of exposition can be regulated to control in these 
systems the rate of polymerization and the conversion degree. The long time of microwaves exposition could enhance the rate of secondary reactions of bond breaking on the pending chains breaking bonds by free radical mechanisms, which would be competitive with the main curing reaction. Properties like hardness, transverse strength, flexural strength, tensile bond strength, and modulus of elasticity, in some studies, are not modified by the longer sample exposition time or by the microwave power, probably because these secondary reactions do not change the cross-link density of the material. Only the impact strength would feel their effect because of the shortening of the pendant chains. The reduction of the impact strength for longer molecules was clearly stated, as well as the influence of the length of the crosslinking agent on the resin mechanical properties (Caycik \& Jagger, 1992).

Researchers investigated mechanical properties of microwave polymerized resins and showed that acrylic resin processed by microwave energy presented the same characteristics of conventional procedures of processing (Nishii, 1968; Stafford \& Handley, 1975; Stafford \& Huggett, 1978; Faraj \& Ellis, 1979; Gourdinne et al., 1979; Kimuta et al., 1983; Reitz et al., 1985; Hayden, 1986; De Clerck, 1987; Kimura et al., 1987; Truong \& Thomasz, 1988; Al-Doori et al., 1988; Al-Mulla et al., 1988; Shlosberg et al., 1989; Levin et al., 1989; Alkhatib et al., 1990; Hogan \& Mori, 1990; Al-Hanbali et al., 1991; Smith et al., 1992; Caycik \& Jagger, 1992;. Chen et al., 1993; Frangou et al., 1993; Jacob et al., 1997). Studies on mechanical properties also showed similar or better results of conventional or light cured resins (Table 3).

\begin{tabular}{|c|c|c|c|}
\hline Authors and date & $\begin{array}{l}\text { Materials and } \\
\text { groups }\end{array}$ & $\begin{array}{l}\text { Methods and } \\
\text { measurements }\end{array}$ & $\begin{array}{l}\text { Comments and } \\
\text { conclusions }\end{array}$ \\
\hline Faot et al. (2008) & $\begin{array}{l}\text { Onda Cryl resin. } \\
\text { Microwave } \\
\text { processing: }(3 \text { min at } \\
360 \mathrm{~W}, 4 \text {-min pause, } \\
\text { and } 3 \text { min at } 810 \mathrm{~W} ; \\
\text { and } 6 \text { min at } 630 \mathrm{~W}) .\end{array}$ & $\begin{array}{l}\text { Impact strength: } \\
\text { Charpy method. } \\
\text { Types and morphology } \\
\text { of fractures: all } \\
\text { fragments analyzed in } \\
\text { morphology, crack } \\
\text { propagation angles and } \\
\text { microstructure. }\end{array}$ & $\begin{array}{l}\text { Both polymerization cycles } \\
\text { are adequate to } \\
\text { polymerize the denture } \\
\text { resin studied. }\end{array}$ \\
\hline $\begin{array}{l}\text { Ganzarolli et al. } \\
\text { (2007) }\end{array}$ & $\begin{array}{l}\text { Conventional water } \\
\text { bath polymerized } \\
\text { resin. } \\
\text { Microwave } \\
\text { polymerized resin. } \\
\text { Injection-molded } \\
\text { resins. }\end{array}$ & $\begin{array}{l}\text { Transverse strength test } \\
\text { - universal machine } \\
\text { under axial load, at a } \\
\text { crosshead speed of } 5 \\
\text { mm/min. } \\
\text { Impact strength test - } \\
\text { Charpy's test } \\
\text { performed with a } 40 \\
\mathrm{~kJ} / \mathrm{cm} \text { load. }\end{array}$ & $\begin{array}{l}\text { There was no relevant } \\
\text { improvement of transverse } \\
\text { and impact strength. } \\
\text { Microwaveable resin } \\
\text { showed similar transverse } \\
\text { and impact strengths. }\end{array}$ \\
\hline $\begin{array}{l}\text { Souza Jr. et al. } \\
\text { (2006) }\end{array}$ & $\begin{array}{l}\text { Acrylic resins } \\
\text { samples: microwave- } \\
\text { (Onda Cryl); visible } \\
\text { light- (Triad); water } \\
\text { bath polymerized } \\
\text { (Clássico). } \\
\text { Cobalt-chromium } \\
\text { metal bar included in } \\
\text { resin samples. }\end{array}$ & $\begin{array}{l}\text { Roughness - } \\
\text { profilometer } \\
\text { (Surfcorder SE 1700) } \\
\text { Knoop hardness - } \\
\left(\mathrm{Kg} / \mathrm{mm}^{2}\right) \text { - } \\
\text { microhardness tester } \\
\text { (Shimadzu HMV } \\
\text { 2000) }\end{array}$ & $\begin{array}{l}\text { The presence of metal did } \\
\text { not influence roughness } \\
\text { and hardness values of any } \\
\text { of the tested acrylic resins }\end{array}$ \\
\hline
\end{tabular}




\begin{tabular}{|c|c|c|c|}
\hline Authors and date & $\begin{array}{c}\text { Materials and } \\
\text { groups } \\
\end{array}$ & $\begin{array}{c}\text { Methods and } \\
\text { measurements }\end{array}$ & $\begin{array}{c}\begin{array}{c}\text { Comments and } \\
\text { conclusions }\end{array} \\
\end{array}$ \\
\hline Tacir et al. (2006) & $\begin{array}{l}\text { PMMA conventional } \\
\text { acrylic resin. } \\
\text { Reinforcing effect of } \\
\text { glass fibres. }\end{array}$ & $\begin{array}{l}\text { Fracture resistance and } \\
\text { Flexural strength. } \\
\text { 3- points of the } \\
\text { samples. } \\
\text { Universal testing } \\
\text { machine }\end{array}$ & $\begin{array}{l}\text { Flexural strength of heat- } \\
\text { polymerized PMMA } \\
\text { denture resin was } \\
\text { improved after } \\
\text { reinforcement with } \\
\text { glass fibres. }\end{array}$ \\
\hline Sarac et al. (2005) & $\begin{array}{l}\text { Denture base } \\
\text { material (a } \\
\text { conventionally } \\
\text { molded, heat } \\
\text { polymerized resin } \\
\text { [Meliodent, M]; an } \\
\text { injection-molded, } \\
\text { Heat polymerized } \\
\text { resin[SRIvocap, I], } \\
\text { and a microwave } \\
\text { polymerized resin } \\
\text { [Acron MC, A]). } \\
\text { Repaired with an } \\
\text { auto polymerizing } \\
\text { acrylic resin } \\
\text { (Meliodent). } \\
\text { Surfaces treated with } \\
\text { chemical etchants: } \\
\text { acetone (30 s), } \\
\text { methylene chloride } \\
\text { (30 sec), MMA (180 } \\
\text { sec). }\end{array}$ & $\begin{array}{l}\text { Shear bond strength } \\
\text { (MPa): universal testing } \\
\text { machine. }\end{array}$ & $\begin{array}{l}\text { Chemical treatment } \\
\text { showed improvement on } \\
\text { the bond strength of the } \\
\text { base materials. } \\
\text { Microwave-polymerized } \\
\text { acrylic resin showed the } \\
\text { lowest shear bond strength } \\
\text { compared to the control } \\
\text { groups. }\end{array}$ \\
\hline $\begin{array}{l}\text { Vergani et al. } \\
(2005)\end{array}$ & $\begin{array}{l}\text { Heat-polymerized } \\
\text { resin (Lucitone 550) } \\
\text { Autopolymerizing } \\
\text { reline resins: } \\
\text { (Duraliner II, } \\
\text { Kooliner, Ufi Gel } \\
\text { Hard, and Tokuso } \\
\text { Rebase Fast). } \\
\text { Postpolymerization } \\
\text { by microwave energy } \\
(500,550 \text {, or } 650 \mathrm{~W}) \\
\text { for }(3,4, \text { or } 5 \\
\text { minutes) }\end{array}$ & $\begin{array}{l}\text { Flexural strength - load } \\
\text { measurements } \\
\text { (Newtons) / crosshead } \\
\text { speed of } 5 \mathrm{~mm} / \mathrm{min} \\
\text { using a 3-point bending } \\
\text { and span of } 50 \mathrm{~mm} .\end{array}$ & $\begin{array}{l}\text { Microwave } \\
\text { postpolymerization } \\
\text { irradiation can be an } \\
\text { effective method for } \\
\text { increasing the flexural } \\
\text { strength of Duraliner II (at } \\
650 \mathrm{~W} \text { ) and Kooliner (at } \\
550 \mathrm{~W} \text { and } 650 \mathrm{~W} \text { for } 5 \\
\text { minutes). }\end{array}$ \\
\hline Yunus et al. (2005) & $\begin{array}{l}\text { Nylon denture base } \\
\text { material (Lucitone } \\
\text { FRS); conventional } \\
\text { compression- } \\
\text { moulded heat- } \\
\text { polymerized } \\
\text { (Meliodent); } \\
\text { compression- } \\
\text { moulded microwave- }\end{array}$ & $\begin{array}{l}\text { Flexural modulus; } \\
\text { flexural strength: three } \\
\text { point bending test. }\end{array}$ & $\begin{array}{l}\text { Flexural modulus of nylon } \\
\text { was significantly lower } \\
\text { than the three PMMA } \\
\text { polymers } \\
\text { Flexural strength of nylon } \\
\text { was significantly lower } \\
\text { than those of Acron MC } \\
\text { (microwaved) and }\end{array}$ \\
\hline
\end{tabular}




\begin{tabular}{|c|c|c|c|}
\hline Authors and date & $\begin{array}{c}\begin{array}{c}\text { Materials and } \\
\text { groups }\end{array} \\
\end{array}$ & $\begin{array}{l}\text { Methods and } \\
\text { measurements }\end{array}$ & $\begin{array}{c}\text { Comments and } \\
\text { conclusions }\end{array}$ \\
\hline & $\begin{array}{l}\text { polymerized } \\
\text { (Acron MC); injection- } \\
\text { moulded microwave } \\
\text { polymerized } \\
\text { (Lucitone 199) PMMA } \\
\text { polymers. } \\
\text { Water stored at } \\
37^{\circ} \mathrm{C} / 30 \text { day } \\
\text { Disinfectant stored for } \\
24 \mathrm{~h}\end{array}$ & & $\begin{array}{l}\text { comparable with Lucitone } \\
199 \text { (microwaved). }\end{array}$ \\
\hline $\begin{array}{l}\text { Phoenix et al. } \\
(2004)\end{array}$ & $\begin{array}{l}6 \text { commonly used } \\
\text { polymethyl } \\
\text { methacrylate denture } \\
\text { base resins. } \\
\text { ADA Specification } \\
\text { No. } 12 \text {. } \\
\text { Thermal assessments } \\
\text { (differential scanning } \\
\text { calorimetry and } \\
\text { dynamic mechanical } \\
\text { analysis). }\end{array}$ & $\begin{array}{l}\text { Color stability, flexural } \\
\text { stiffness, and hardness. }\end{array}$ & $\begin{array}{l}\text { The microwaveable resins } \\
\text { displayed greater stiffness, } \\
\text { and greater surface } \\
\text { hardness than other } \\
\text { denture base resins. } \\
\text { Elastomeric toughening } \\
\text { agents yielded decreased } \\
\text { stiffness, decreased surface } \\
\text { hardness, and decreased } \\
\text { glass transition } \\
\text { temperatures. }\end{array}$ \\
\hline Lai et al. (2004) & $\begin{array}{l}\text { PMMA denture base } \\
\text { polymer (Optilon- } \\
399) \text { - (water-bath at } \\
70^{\circ} \mathrm{C} \text { for } 9 \text { h; resin } \\
\text { blocks was processed } \\
\text { at } 80,160,240 \text {, and } \\
560 \mathrm{~W} \text { for } 15,10,7 \text {, } \\
\text { and } 2 \text { min, separately } \\
+ \text { additional } 2 \text { min at } \\
560 \text { W. } \\
\text { Thickness samples } \\
\text { were less than } 10 \\
\text { mm. }\end{array}$ & $\begin{array}{l}\text { Hardness - Shimadzu } \\
\text { hardness tester (HMV- } \\
2000-300 \mathrm{~g} / 30 \mathrm{~s}-15 \\
\text { areas along uniformly } \\
\text { selected points of } \\
\text { surfaces). } \\
\text { Flexural strength - MTS } \\
\text { dynamic tensile testing } \\
\text { machine (Model } 810 \text { ) at } \\
\text { a crosshead speed of } \\
1.25 \text { mm/min. Three- } \\
\text { point-bending test. }\end{array}$ & $\begin{array}{l}\text { Highly statistical } \\
\text { differences in flexural } \\
\text { properties were evident in } \\
\text { a comparison of } \\
\text { processing methods. } \\
\text { The size and the volume } \\
\text { fraction of the rubber } \\
\text { phase are in favor of the } \\
\text { water-bath method. } \\
\text { Water-bath cured } \\
\text { specimens showed better } \\
\text { flexural strength and } \\
\text { fexural modulus than the } \\
\text { microwave-cured } \\
\text { specimens. There were no } \\
\text { significant differences in } \\
\text { the surface hardness and } \\
\text { the domain size } \\
\text { distribution of the effective } \\
\text { rubber phase. }\end{array}$ \\
\hline $\begin{array}{l}\text { Oliveira et al. } \\
(2003)\end{array}$ & $\begin{array}{l}\text { Acron MC - } \\
500 \mathrm{~W} / 3 \mathrm{~m} \text { or } 4.5 \mathrm{~m} \\
\text { One simple flask } \\
\text { centrally placed on } \\
\text { the turning plate; two } \\
\text { flasks, one in the } \\
\text { centre and the other } \\
\text { peripherally placed in } \\
\text { the plate; two flasks } \\
\text { centrally, one above } \\
\text { and the other below. }\end{array}$ & $\begin{array}{l}\text { Hardness test - } 12 \\
\text { indentations in the } \\
\text { surface of specimen. }\end{array}$ & $\begin{array}{l}\text { There is no difference } \\
\text { among the groups. }\end{array}$ \\
\hline
\end{tabular}




\begin{tabular}{|c|c|c|c|}
\hline Authors and date & $\begin{array}{c}\text { Materials and } \\
\text { groups }\end{array}$ & $\begin{array}{c}\text { Methods and } \\
\text { measurements }\end{array}$ & $\begin{array}{c}\text { Comments and } \\
\text { conclusions }\end{array}$ \\
\hline $\begin{array}{l}\text { Azzarri et al. } \\
(2003)\end{array}$ & $\begin{array}{l}\text { Acrylic denture base } \\
\text { resin microwave } \\
\text { polymerized } \\
\text { (samples were } \\
\text { prepared in } 200,500, \\
\text { and } 800, \text { for } 5 \text { and } 10 \\
\text { min each side up). }\end{array}$ & $\begin{array}{l}\text { Hardness ( Rockwell } \\
\text { method) Strength } \\
\text { (Charpy method). } \\
\text { Young's modulus of } \\
\text { elasticity - technique } \\
\text { described by Stafford \& } \\
\text { Handley (1975) } \\
\text { according to ISO } 1567 .\end{array}$ & $\begin{array}{l}\text { The mechanical properties } \\
\text { of the acrylic denture base } \\
\text { resin microwave } \\
\text { polymerized depend both } \\
\text { on the exposition time and } \\
\text { microwave power. } \\
\text { From the appropriate } \\
\text { selection of power and } \\
\text { time of curing of the resin } \\
\text { it is possible to obtain the } \\
\text { best mechanical properties. }\end{array}$ \\
\hline $\begin{array}{l}\text { Schneider et al. } \\
\text { (2002) }\end{array}$ & $\begin{array}{l}\text { Acrylic resins } \\
\text { samples: heat } \\
\text { polymerized } \\
\text { (Lucitone 199); and } \\
\text { Microwave } \\
\text { polymerized (Acron } \\
\text { MC). } \\
\text { Acrylic resin denture } \\
\text { teeth: (IPN, SLM, } \\
\text { Vitapan, and } \\
\text { SROrthotyp-PE). } \\
\text { ADA specification } \\
\mathrm{n}^{\circ} 15 .\end{array}$ & $\begin{array}{l}\text { Strength until sample } \\
\text { fracture - custom } \\
\text { alignment device } \\
\text { debonded specimens. } \\
\text { Cohesive failures - } \\
\text { scanning electron } \\
\text { microscope. }\end{array}$ & $\begin{array}{l}\text { Type of denture base } \\
\text { material and denture tooth } \\
\text { selected for use may } \\
\text { influence the tensile bond } \\
\text { strength of the tooth to the } \\
\text { base. }\end{array}$ \\
\hline $\begin{array}{l}\text { Memon et al. } \\
\text { (2001) }\end{array}$ & $\begin{array}{l}\text { Microwave } \\
\text { polymerized } \\
\text { polyurethane-based } \\
\text { denture material } \\
\text { processed by an } \\
\text { injection-molding } \\
\text { technique, a } \\
\text { conventional } \\
\text { microwave } \\
\text { polymerized denture } \\
\text { material, and a heat } \\
\text { polymerized } \\
\text { compression-molded } \\
\text { poly(methyl } \\
\text { methacrylate) } \\
\text { (PMMA) denture } \\
\text { material. }\end{array}$ & $\begin{array}{l}\text { Impact strength - } \\
\text { Charpy-type impact } \\
\text { tester. } \\
\text { Transverse strength } \\
\text { and the flexural } \\
\text { modulus - three-point } \\
\text { bending test. }\end{array}$ & $\begin{array}{l}\text { Impact and flexural } \\
\text { strengths - microwave } \\
\text { polymerized injection } \\
\text { molded, offered no } \\
\text { advantage over the } \\
\text { existing heat- and } \\
\text { microwave-polymerized } \\
\text { PMMA-based denture } \\
\text { base polymer. } \\
\text { It has rigidity comparable } \\
\text { to that of the microwave- } \\
\text { polymerized PMMA } \\
\text { polymer. }\end{array}$ \\
\hline $\begin{array}{l}\text { Polyzois et al. } \\
\text { (2001) }\end{array}$ & $\begin{array}{l}\text { Heat-polymerized } \\
\text { denture base material } \\
\text { repaired with heat } \\
\text { polymerized resin. } \\
\text { Auto polymerized } \\
\text { resin alone. } \\
\text { Auto polymerized } \\
\text { resin with glass fiber }\end{array}$ & $\begin{array}{l}\text { Fracture force, } \\
\text { deflection at fracture, } \\
\text { toughness: 3-point } \\
\text { bending test. }\end{array}$ & $\begin{array}{l}\text { The most effective was } \\
\text { microwave-irradiated, } \\
\text { auto polymerized resin } \\
\text { reinforced with round } \\
\text { wire or monolayer glass } \\
\text { fiber ribbon. }\end{array}$ \\
\hline
\end{tabular}




\begin{tabular}{|c|c|c|c|}
\hline Authors and date & $\begin{array}{c}\text { Materials and } \\
\text { groups }\end{array}$ & $\begin{array}{l}\text { Methods and } \\
\text { measurements }\end{array}$ & $\begin{array}{c}\text { Comments and } \\
\text { conclusions }\end{array}$ \\
\hline & $\begin{array}{l}\text { or wire } \\
\text { reinforcement. } \\
\text { Auto polymerized } \\
\text { resin repairs; heat } \\
\text { polymerized resin } \\
\text { repairs (with no } \\
\text { reinforcement, } \\
\text { treated with } \\
\text { microwave } \\
\text { irradiation after } \\
\text { polymerization, with } \\
\text { monolayer or } \\
\text { multilayer glass fiber } \\
\text { reinforcement, and } \\
\text { with round or } \\
\text { braided wire } \\
\text { reinforcement). }\end{array}$ & & \\
\hline $\begin{array}{l}\text { Rached et al. } \\
\text { (2001) }\end{array}$ & $\begin{array}{l}\text { Heat-cured acrylic } \\
\text { resin (Lucitone 550) - } \\
\text { repaired with a } \\
\text { microwave acrylic } \\
\text { resin (Acron MC) - } \\
500 \mathrm{~W} / 3 \mathrm{~m} \text {. } \\
\text { Chemical treatments } \\
\text { (AC monomer } \\
\text { dipping/30 s; acetone } \\
\text { dipping/30 s; acetone } \\
\text { dipping/15 s + blast } \\
\text { of air + AC monomer } \\
\text { dipping/15 s) in the } \\
\text { cut ends. }\end{array}$ & $\begin{array}{l}\text { Surface texture - } \\
\text { scanning electron } \\
\text { microscopy. } \\
\text { Flexural strengh. }\end{array}$ & $\begin{array}{l}\text { Surface treatments affected } \\
\text { the bond strength between } \\
\text { the two acrylic resins. } \\
\text { There are no differences in } \\
\text { strength between intact } \\
\text { heat-cured denture base } \\
\text { material and the same } \\
\text { material repaired with } \\
\text { microwave acrylic resin. }\end{array}$ \\
\hline $\begin{array}{l}\text { Blabojevic et al. } \\
\text { (1999) }\end{array}$ & $\begin{array}{l}\text { A without } \\
\text { crosslinking agents } \\
\text { homopolymer } \\
\text { (TS1195). } \\
\text { A cross-linked } \\
\text { microwave resin } \\
\text { (Acron MC) } \\
\text { (Microwave } \\
\text { Panasonic oven with } \\
\text { rotating turntable). } \\
\text { A cross-linked for } \\
\text { water-bath } \\
\text { polymerization } \\
\text { (Biocryl NR) } \\
\text { (Conventional water } \\
\text { bath). } \\
\text { An autopolymerizing } \\
\text { reline:repair material } \\
\text { (CroForm). }\end{array}$ & $\begin{array}{l}\text { Indentation hardness - } \\
\text { Wallace hardness tester } \\
\text { Model H6B:SA:C } \\
\text { Impact strength - } \\
\text { Zwick pendulum } \\
\text { impact model } 5102 \text { with } \\
0.5 \text { J pendulum. }\end{array}$ & $\begin{array}{l}\text { Microwaving of } \\
\text { autopolymerizing resin } \\
\text { improved analyzed } \\
\text { mechanical properties. }\end{array}$ \\
\hline
\end{tabular}




\begin{tabular}{|c|c|c|c|}
\hline Authors and date & $\begin{array}{c}\begin{array}{c}\text { Materials and } \\
\text { groups }\end{array} \\
\end{array}$ & $\begin{array}{c}\text { Methods and } \\
\text { measurements }\end{array}$ & $\begin{array}{c}\text { Comments and } \\
\text { conclusions }\end{array}$ \\
\hline $\begin{array}{l}\text { Polyzois et al. } \\
\text { (1995) }\end{array}$ & $\begin{array}{l}\text { Denture base resins } \\
\text { repaired (standard } \\
\text { heat activated } \\
\text { polymerising resin, a } \\
\text { denture base resin } \\
\text { especially formulated } \\
\text { for microwave } \\
\text { activated } \\
\text { polymerisation, } \\
\text { auto polymerizing } \\
\text { resin). } \\
\text { Conventional water } \\
\text { bath; microwave } \\
\text { curing, and auto } \\
\text { polymerized resin } \\
\text { repairs. }\end{array}$ & $\begin{array}{l}\text { Transverse Bend } \\
\text { impact tests }\end{array}$ & $\begin{array}{l}\text { The transverse strength, } \\
\text { and impact resistence of } \\
\text { the resin specimens } \\
\text { repaired with microwave } \\
\text { irradiation were generally } \\
\text { superior to specimens } \\
\text { repaired by using a water } \\
\text { bath curing cycle or the } \\
\text { use of an } \\
\text { autopolymerising resin. }\end{array}$ \\
\hline Yunus et al. (1994) & $\begin{array}{l}\text { Acrylic resin } \\
\text { repair material } \\
\text { bench-cure, } \\
\text { hydroflask-cure and } \\
\text { microwave } \\
\text { irradiation cure. } \\
\end{array}$ & Repair strength. & $\begin{array}{l}\text { On the three methods, } \\
\text { polymerization using } \\
\text { microwave energy } \\
\text { resulted in the strongest } \\
\text { repair. }\end{array}$ \\
\hline Ilbay et al. (1994) & $\begin{array}{l}\text { Twenty-one different } \\
\text { polymerization } \\
\text { methods were used } \\
\text { by varying radiation } \\
\text { power and curing } \\
\text { time. } \\
(3 \text { min at } 550 \mathrm{~W})\end{array}$ & $\begin{array}{l}\text { Vickers hardness test } \\
\text { transverse load } \\
\text { transverse deflection }\end{array}$ & $\begin{array}{l}\text { Resin cured by microwave } \\
\text { energy is more resistant to } \\
\text { mechanical failure than } \\
\text { conventionally cured } \\
\text { acrylic. }\end{array}$ \\
\hline $\begin{array}{l}\text { Del bel Cury et al. } \\
\text { (1994) }\end{array}$ & $\begin{array}{l}\text { Acrylic resins: Acron } \\
\mathrm{MC} \text { (microwave - } \\
500 \mathrm{~W} / 3 \mathrm{~m}) ; \text { Lucitone } \\
550 \text { (water bath - } \\
\left.73^{\circ} \mathrm{C} / 90 \mathrm{~m}\right) ; \text { Ortho- } \\
\text { Class (self } \\
\text { polymerized). }\end{array}$ & $\begin{array}{l}\text { Transverse strength } \\
\text { and maximum } \\
\text { deflection - assay } \\
\text { machine in three points } \\
\text { (Instron } 125) 5 \mathrm{~mm} / \mathrm{m} \text {. } \\
\text { Impact - Charpy assay. }\end{array}$ & $\begin{array}{l}\text { There were differences } \\
\text { among tested materials } \\
\text { that can be resulted from } \\
\text { composition and } \\
\text { polymerization methods. }\end{array}$ \\
\hline
\end{tabular}

Table 3. Studies on hardness, transverse strength, flexural strength, shear bond strength, tensile bond strength, impact strength, roughness, and modulus of elasticity of microwaved resin.

\section{Base adaptation, dimensional alteration, artificial tooth movement, and teeth occlusion}

Considering dimensional alteration of denture or bases resin, when conventional water bath and microwave energy were compared, some authors (Reitz et al., 1985; Levin et al., 1989; Uchida et al., 1989; Takamata et al., 1989; Al-Hanbali et al., 1991; Nelson et al., 1991; Wallace et al., 1991; Sanders et al., 1991; Barbosa et al., 2002; Keenan et al., 2003) found no difference between the two techniques; also, these results are not in agreement with others (Sanders et al., 1991; Nelson et al, 1991). Sanders et al. (1991) observed in their study that microwave 
polymerization provided a lower degree of artificial tooth movement, while Nelson et al (1991) reported a greater degree of tooth positional changes when microwave polymerization was employed. Although different investing mediums or polymerization techniques, have been compared (Reitzet al., 1985; Levin et al. 1989; Nelson et al., 1991; Turck \& Richards, 1992) the authors could not identify studies published concerning the outcomes provided by the combination of different flasking methods and polymerization techniques. Dimensional changes and distortion of the denture due to the investing stone mold and the heating of acrylic resin can promote tooth movement and, consequently alterations in the occlusal contacts and occlusal vertical dimension (OVD). (Rizzatti-Barbosa et al., 2006; Rizzatti-Barbosa et al., 2005). Acrylic resin processing methods do not avoid displacement of artificial teeth during denture inclusion and processing, which might increase occlusal vertical dimension due to production of premature contacts (Barbosa et al., 2002). It is hence necessary to adjust the oclusal surface of artificial teeth, which alters the occlusal anatomy, especially of posterior teeth (Lai et al., 2004). In addition, these alterations may cause mucosal injuries and affect the functionality of the prostheses, thus causing damage to the stomatognathic system, temporomandibular disorders and discomfort to the patient (Yagi et al., 2006). Simultaneous polymerization of maxillary and mandibular complete dentures with the teeth in occlusion by means of a special double flask (DF), has been described as a more rapid and simple method for investing and polymerizing prostheses (Rizzatti-Barbosa et al., 2005). This inclusion technique was claimed to save time and decrease occlusal alteration during denture processing (Meloto et al., 2006). It may be an easier and faster method of investing and polymerizing prostheses. The first designed DF was a metal copper-aluminum flask (DMF) for simultaneous polymerization of both maxillary and mandibular prostheses in a warm water bath (Dental VIPI Ltd, Pirassununga, Brazil). The double polyvinyl chloride flask (DPVCF) (Dental VIPI Ltd, Pirassununga, Brazil) was developed following the same principles for simultaneous processing of both dentures in occlusion through microwave energy heating (Rizzatti-Barbosa et al., 2005). This new technique associating acrylic curing with microwave energy can be considered a clean method that saves time, reduces occlusal interferences, preserves the teeth occlusion, and maintains the OVD. (Rizzatti-Barbosa \& Ribeiro-Dasilva, 2009). In the classical literature, some data on the acrylic resins morphology alteration can be found (Huggett et al., 1984; Polyzois et al., 1987; Chen et al., 1988; Jagger, 1996). Some controversial results about microwave processing resin are shown on table 4 , where the authors compared microwaving technique with other and related the results of teeth positioning, dimensional alteration, vertical measurement of dentures, etc.

\begin{tabular}{|c|c|c|c|}
\hline Authors and date & Materials and groups & $\begin{array}{l}\text { Methods and } \\
\text { measurements }\end{array}$ & $\begin{array}{l}\text { Comments and } \\
\text { conclusions }\end{array}$ \\
\hline $\begin{array}{l}\text { Negreiros et al. } \\
(2009)\end{array}$ & $\begin{array}{l}\text { Clássico (Conventional } \\
\text { long cycle in water } \\
\text { bath). } \\
\text { Onda-Cryl (Microwave } \\
\text { energy). } \\
\text { QC-20 (Fast cycle in } \\
\text { boiling water). } \\
\text { Post pressing process. }\end{array}$ & $\begin{array}{l}\text { The linear distances - } \\
\text { STM microscope (right } \\
\text { premolar to left } \\
\text { premolar; right molar to } \\
\text { left molar; right incisor } \\
\text { to right molar; left } \\
\text { incisor to left molar). }\end{array}$ & $\begin{array}{l}\text { Microwave } \\
\text { polymerization was } \\
\text { similar to that of the } \\
\text { conventional cycles } \\
\text { in water bath } \\
\text { post-pressing time } \\
\text { had no relevant } \\
\text { effect on tooth } \\
\text { movement. }\end{array}$ \\
\hline
\end{tabular}




\begin{tabular}{|c|c|c|c|}
\hline Authors and date & Materials and groups & $\begin{array}{l}\text { Methods and } \\
\text { measurements }\end{array}$ & $\begin{array}{l}\text { Comments and } \\
\text { conclusions }\end{array}$ \\
\hline $\begin{array}{l}\text { Schibayama et al. } \\
\text { (2009) }\end{array}$ & $\begin{array}{l}\text { Dentures processed with: } \\
\text { Acrylic resin for water } \\
\text { bath polymerization } \\
\text { (QC20); Acrylic resin for } \\
\text { microwave } \\
\text { polymerization (Acron } \\
\text { MC). } \\
\text { Flasking: (1) adding a } \\
\text { second investment layer } \\
\text { of gypsum and } \\
\text { conventional water bath } \\
\text { polymerization, (2) } \\
\text { adding a second } \\
\text { investment layer of } \\
\text { gypsum and } \\
\text { polymerization with } \\
\text { microwave energy, (3) } \\
\text { adding a second } \\
\text { investment layer of } \\
\text { silicone and } \\
\text { conventional } \\
\text { polymerization, and (4) } \\
\text { adding a second } \\
\text { investment layer of } \\
\text { silicone and } \\
\text { polymerization with } \\
\text { microwave energy. }\end{array}$ & $\begin{array}{l}\text { Comparison of the } \\
\text { artificial tooth position } \\
\text { changes following } \\
\text { flasking and } \\
\text { polymerization of } \\
\text { dentures - linear } \\
\text { microscope. }\end{array}$ & $\begin{array}{l}\text { The use of a silicone } \\
\text { investment layer } \\
\text { when flasking } \\
\text { complete dentures } \\
\text { resulted in the least } \\
\text { positional changes } \\
\text { of the artificial teeth } \\
\text { regardless of the } \\
\text { polymerization } \\
\text { technique. }\end{array}$ \\
\hline Faot et al. (2008) & $\begin{array}{l}\text { Onda Cryl resin } \\
\text { Microwave processing: } \\
(3 \mathrm{~min} \text { at } 360 \mathrm{~W}, 4-\mathrm{min} . \\
\text { pause, and } 3 \mathrm{~min} \text { at } 810 \\
\mathrm{~W} \text {; and } 6 \text { min at } 630 \mathrm{~W}) .\end{array}$ & $\begin{array}{l}\text { Accuracy of fit: } 3 \text { points } \\
\text { at the right and left ridge } \\
\text { crests and at the midline } \\
\text { on the posterior palatal } \\
\text { seal for each denture } \\
\text { base. }\end{array}$ & $\begin{array}{l}\text { Both polymerization } \\
\text { cycles are adequate } \\
\text { to polymerize the } \\
\text { studied denture } \\
\text { resin. }\end{array}$ \\
\hline Pavan et al. (2005) & $\begin{array}{l}\text { Dentures in acrylic resin } \\
\text { (Clássico - water bath } \\
\text { processing). } \\
\text { Storage in water for } 30 \\
\text { days. } \\
\text { Microwave disinfection: } \\
3 \text { min at } 500 \mathrm{~W} \text {; and } 10 \\
\text { min at } 604 \mathrm{~W} \text {. }\end{array}$ & $\begin{array}{l}\text { Dimensional accuracy } \\
\text { along the posterior } \\
\text { palatal border of } \\
\text { maxillary acrylic resin } \\
\text { denture bases. }\end{array}$ & $\begin{array}{l}\text { Treatment in } \\
\text { microwave oven at } \\
604 \mathrm{~W} \text { for } 10 \mathrm{~min} \\
\text { produced the } \\
\text { greatest } \\
\text { discrepancies in the } \\
\text { adaptation of } \\
\text { maxillary acrylic } \\
\text { resin denture bases } \\
\text { to the stone casts. }\end{array}$ \\
\hline $\begin{array}{l}\text { Rizzatti-Barbosa et } \\
\text { al. (2005) }\end{array}$ & $\begin{array}{l}\text { Pairs of dentures } \\
\text { processed by microwave } \\
\text { energy and flasked in } \\
\text { occlusion in double } \\
\text { flasks. }\end{array}$ & $\begin{array}{l}\text { Occlusal inclination of } \\
\text { mandibular and } \\
\text { maxillary artificial } \\
\text { denture molars. }\end{array}$ & $\begin{array}{l}\text { Dentures double } \\
\text { flasking and } \\
\text { microwave curing } \\
\text { save time, reduce } \\
\text { occlusal alteration, } \\
\text { and reduce time } \\
\text { exposure of } \\
\text { dentures. }\end{array}$ \\
\hline
\end{tabular}




\begin{tabular}{|c|c|c|c|}
\hline Authors and date & Materials and groups & $\begin{array}{c}\text { Methods and } \\
\text { measurements }\end{array}$ & $\begin{array}{c}\text { Comments and } \\
\text { conclusions }\end{array}$ \\
\hline Lai et al. (2004) & $\begin{array}{l}\text { Microwaved resin } \\
\text { blocks, processed at } 80 \text {, } \\
160,240 \text {, and } 560 \mathrm{~W} \text { for } \\
15,10,7 \text {, and } 2 \mathrm{~min} / \\
70^{\circ} \mathrm{C} \text { for } 9 \mathrm{~h} .\end{array}$ & $\begin{array}{l}\text { The morphology of the } \\
\text { specimens after staining } \\
\text { with osmium tetroxide } \\
\text { was examined by } \\
\text { transmission electron } \\
\text { microscope. }\end{array}$ & $\begin{array}{l}\text { Highly statistical } \\
\text { differences in } \\
\text { morphology favor } \\
\text { of the water-bath } \\
\text { method. }\end{array}$ \\
\hline Botega et al. (2004) & $\begin{array}{l}\text { Retangular samples and } \\
\text { half-discs of } \\
\text { microwaved-cure acrylic } \\
\text { resin (Acron MC). } \\
1,2,4 \text {, and } 6 \text { flasks } \\
\text { simultaneous } \\
\text { processing. } \\
\text { Polymerization time } \\
\text { starting in 3min/450W - } \\
\text { adjustmentsbased on } \\
\text { monomer release. }\end{array}$ & $\begin{array}{l}\text { Monomer release: } \\
\text { spectrophotometer } \\
\text { Beckman DU-70. } \\
\text { Knoop hardness: } \\
\text { Shimadzu HMV } 2000 \\
\text { hardness tester. } \\
\text { Porosity: black inch } \\
\text { immersion and count } \\
\text { pores in microscope. }\end{array}$ & $\begin{array}{l}\text { The number of } \\
\text { flasks does not } \\
\text { interfere with } \\
\text { polymerization, } \\
\text { knoop hardness, } \\
\text { and porosity. }\end{array}$ \\
\hline Phoenix et al. (2004) & $\begin{array}{l}6 \text { commonly used } \\
\text { polymethyl } \\
\text { methacrylate denture } \\
\text { base resins. ADA } \\
\text { Specification No. } 12 . \\
\text { Thermal assessments } \\
\text { (differential scanning } \\
\text { calorimetry and } \\
\text { dynamic mechanical } \\
\text { analysis). }\end{array}$ & $\begin{array}{l}\text { Adaptation denture } \\
\text { bases. }\end{array}$ & $\begin{array}{l}\text { The microwaveable } \\
\text { resins displayed } \\
\text { better adaptation } \\
\text { than other denture } \\
\text { base resins included } \\
\text { in this investigation. }\end{array}$ \\
\hline Keenan et al. (2003) & $\begin{array}{l}\text { Identical maxillary } \\
\text { denture bases in: PMMA } \\
\text { (Trevalon - Compression } \\
\text { flask - hot air oven); } \\
\text { microwave polymerized } \\
\text { resin (AcronMC } \\
\text { Injection flask - 600-W } \\
\text { microwave oven/3m); } \\
\text { PMMA (Trevalon - } \\
\text { Injection flask - hot air } \\
\text { oven); resin injection } \\
\text { flasks (Microbase } \\
\text { Injection flask - 600-W } \\
\text { microwave oven/ 6m) }\end{array}$ & $\begin{array}{l}\text { Pre and post treatment } \\
\text { intermolar width - } \\
\text { traveling microscope. } \\
\text { Pre and post treatment } \\
\text { vertical dimension of } \\
\text { occlusion - points on the } \\
\text { superior and inferior } \\
\text { members of the } \\
\text { articulator. }\end{array}$ & $\begin{array}{l}\text { All injection } \\
\text { molding methods } \\
\text { produced dentures } \\
\text { with a slightly } \\
\text { smaller increase in } \\
\text { vertical dimension } \\
\text { of occlusion. } \\
\text { Both microwave } \\
\text { polymerization } \\
\text { methods produced } \\
\text { maxillary complete } \\
\text { dentures with a } \\
\text { greater reduction in } \\
\text { intermolar width. }\end{array}$ \\
\hline $\begin{array}{l}\text { Ganzarolli et al. } \\
\text { (2002) }\end{array}$ & $\begin{array}{l}\text { Heat-cured acrylic resin } \\
\text { (Classico); two } \\
\text { microwave-cured acrylic } \\
\text { resins (Acron MC and } \\
\text { Onda Cryl). } \\
\text { Water storage. }\end{array}$ & $\begin{array}{l}\text { Adaptation - weight of } \\
\text { silicone impression } \\
\text { material between the } \\
\text { base and the master die. }\end{array}$ & $\begin{array}{l}\text { Interaction of type } \\
\text { of material and } \\
\text { cooling procedure } \\
\text { has effect on the } \\
\text { final adaptation. } \\
\text { Water storage was } \\
\text { not a source of } \\
\text { variance on the final } \\
\text { adaptation. }\end{array}$ \\
\hline
\end{tabular}




\begin{tabular}{|c|c|c|c|}
\hline Authors and date & Materials and groups & $\begin{array}{l}\text { Methods and } \\
\text { measurements }\end{array}$ & $\begin{array}{l}\text { Comments and } \\
\text { conclusions }\end{array}$ \\
\hline Barbosa et al. (2002) & $\begin{array}{l}\text { Maxillary dentures } \\
\text { polymerization with } \\
\text { different cycles by } \\
\text { microwave radiation. }\end{array}$ & $\begin{array}{l}\text { Changes in occlusal } \\
\text { vertical dimension: } \\
\text { average in articulator } \\
\text { pin opening }\end{array}$ & $\begin{array}{l}\text { There was no } \\
\text { difference between } \\
\text { the groups } \\
\text { polymerized by the } \\
\text { microwave method } \\
\text { and the control } \\
\text { group }\end{array}$ \\
\hline $\begin{array}{l}\text { Del Bel Cury et al. } \\
\text { (2001) }\end{array}$ & $\begin{array}{l}\text { Two microwave-cured } \\
\text { acrylic resins (Acron MC } \\
\text { and Onda Cryl). } \\
\text { Gypsum moulding } \\
\text { technique or silicone } \\
\text { gypsum moulding } \\
\text { technique. }\end{array}$ & $\begin{array}{l}\text { Residual monomer - } 24 \\
\text { or } 48 \mathrm{~h} \text { over a period of } \\
288 \mathrm{~h} \text {. } \\
\text { Knoop hardness - after } \\
24,48,72 \mathrm{~h} \text { and } 30 \text { days. } \\
\text { Transverse strength - } 48 \\
\text { h of water storage. }\end{array}$ & $\begin{array}{l}\text { Storage periods and } \\
\text { moulding technique } \\
\text { did not influence } \\
\text { Knoop hardness. } \\
\text { The type of mould } \\
\text { did not affect } \\
\text { tranverse strength. } \\
\text { The acrylic resins } \\
\text { differed from each } \\
\text { other for all } \\
\text { properties, } \\
\text { regardless of the } \\
\text { type of mould. }\end{array}$ \\
\hline Braun et al. (2000) & $\begin{array}{l}\text { Samples of acrylic resins } \\
\text { (Classico, Lucitone 550, } \\
\text { Acron MC). } \\
\text { Water bath (long cicle), } \\
\text { water bath (short cicle), } \\
\text { and microwave energy } \\
\text { (500W/3 m). } \\
\text { Water sorption - } 30 \text { days. }\end{array}$ & $\begin{array}{l}\text { Linear dimensional } \\
\text { alteration - pre and post } \\
\text { water sorption period } \\
\text { (linear microscopy). }\end{array}$ & $\begin{array}{l}\text { All samples } \\
\text { presented expansion } \\
\text { after water sorption } \\
\text { period. }\end{array}$ \\
\hline Wong et al. (1999) & $\begin{array}{l}\text { Dentures polymerization } \\
\text { by } 3 \text { processing } \\
\text { techniques (dry and wet } \\
\text { heat; different rates of } \\
\text { cooling). }\end{array}$ & $\begin{array}{l}\text { Dimensional changes - } \\
\text { traveling microscope. } \\
\text { Water sorption - } \\
\text { electronic balance (water } \\
\text { uptake). }\end{array}$ & $\begin{array}{l}\text { Water uptake after } \\
\text { deflasking was low. } \\
\text { The dentures did not } \\
\text { reveal differences in } \\
\text { shrinkage at water } \\
\text { saturation. } \\
\text { Oven-processed and } \\
\text { water bath processed } \\
\text { acrylic resin } \\
\text { dentures showed } \\
\text { similar dimensional } \\
\text { shrinkage at water } \\
\text { saturation. }\end{array}$ \\
\hline $\begin{array}{l}\text { Sadamori et al. } \\
\text { (1997) }\end{array}$ & $\begin{array}{l}\text { Acrylic resin dentures. } \\
\text { Different thickness. } \\
\text { Processing: conventional } \\
\text { method, fluid resin } \\
\text { technique, and } \\
\text { microwave curing } \\
\text { method. }\end{array}$ & $\begin{array}{l}\text { Residual monomer - gas } \\
\text { liquid chromatography. } \\
\text { Dimensional accuracy, } \\
\text { and resin stability. }\end{array}$ & $\begin{array}{l}\text { Dimensional } \\
\text { accuracy and } \\
\text { stability of acrylic } \\
\text { resin dentures could } \\
\text { be influenced by the } \\
\text { processing method. } \\
\text { The thickness of the } \\
\text { bases, and the shape } \\
\text { and size of the } \\
\text { dentures influenced } \\
\text { on dimensional } \\
\text { changes. }\end{array}$ \\
\hline
\end{tabular}




\begin{tabular}{|c|c|c|c|}
\hline Authors and date & Materials and groups & $\begin{array}{c}\text { Methods and } \\
\text { measurements }\end{array}$ & $\begin{array}{c}\text { Comments and } \\
\text { conclusions }\end{array}$ \\
\hline $\begin{array}{l}\text { Rodrigues Garcia et } \\
\text { al. (1996) }\end{array}$ & $\begin{array}{l}\text { Denture bases of } \\
\text { conventional acrylic } \\
\text { resin (water bath } 73^{\circ} \mathrm{C} / \\
9 \text { hours) and specific } \\
\text { resin for microwave } \\
\text { polymerization ( } 500 \\
W / 3 \mathrm{~m}) \text {. } \\
\text { Dentures were relined } \\
\text { by addition method. }\end{array}$ & $\begin{array}{l}\text { Accuracy - weight of an } \\
\text { impression material put } \\
\text { between the denture } \\
\text { base and cast die. }\end{array}$ & $\begin{array}{l}\text { Conventional resin } \\
\text { cured by water bath } \\
\text { or microwave } \\
\text { energy showed } \\
\text { better adaptation. }\end{array}$ \\
\hline $\begin{array}{l}\text { Rizzatti-Barbosa et } \\
\text { al. (1995) }\end{array}$ & $\begin{array}{l}\text { Dentures in acrylic resin: } \\
\text { Acron MC (microwaved } \\
-500 \mathrm{~W} / 3 \mathrm{~m}) ; \text { Lucitone } \\
150 \text { (water bath - } \\
\left.72^{\circ} \mathrm{C} / 9 \mathrm{~h}\right) . \\
\text { Water Storage for } 30 \\
\text { days. }\end{array}$ & $\begin{array}{l}\text { Posterior palatal fit } \\
\text { weight and } \\
\text { measurement of } \\
\text { impression material } \\
\text { between the denture } \\
\text { base and master cast. }\end{array}$ & $\begin{array}{l}\text { There were no } \\
\text { difference among } \\
\text { the resins, } \\
\text { polymerization } \\
\text { methods and water } \\
\text { storage period. }\end{array}$ \\
\hline Salim et al. (1992) & $\begin{array}{l}\text { Rectangular acrylic resin } \\
\text { specimens processed by } \\
\text { three methods: a } \\
\text { conventional method, } \\
\text { the SR-Ivocap system, } \\
\text { and a microwave curing } \\
\text { method. }\end{array}$ & $\begin{array}{l}\text { Dimensional accuracy - } \\
\text { change of the distance } \\
\text { vector V (calculated by } \\
\text { means of measurements } \\
\text { of the distances between } \\
\text { fixed points on } \\
\text { specimens). }\end{array}$ & $\begin{array}{l}\text { SR-Ivocap system } \\
\text { exhibited less } \\
\text { dimensional change. } \\
\text { SR- Ivocap system } \\
\text { might produce more } \\
\text { accurate denture } \\
\text { base than } \\
\text { conventional and } \\
\text { microwave curing } \\
\text { methods. }\end{array}$ \\
\hline
\end{tabular}

Table 4. Studies on base adaptation, dimensional alteration, artificial tooth movement, and teeth occlusion of microwaved resin

\section{Studies about the effects of microwave disinfection}

Bacterial and yeast plaque on dentures may lead to serious infections, such as systemic candidal infection, particularly in patients who have debilitating diseases (Montagner et al., 2009). The use of microwave energy to disinfect dentures has been suggested to overcome the problems associated with denture cleaning. Microwave energy was introduced in 1985 for sterilization of nonautoclavable dental materials. It was shown that exposed to microwave energy for 10 minutes can kill microorganisms if the denture is attached to a three-dimensional rotating device (Rohler \& Bulard, 1985).

Lining materials have been found to be more prone to microbial adhesion than acrylic resin base materials and have been demonstrated to interact with oral microorganisms because of their surface texture and the physical/chemical affinity between the materials. Surface roughness of the resilient liners may differ among materials (Zissis et al., 2000; Jin et al., 2003), and rougher surfaces enhance the adhesion of microorganisms onto resilient lining materials (Bulad et al., 2004) that may allow fungal growth (Brosky et al., 2003). The microorganisms from the plaque on the denture surface may expose patients and dental personnel to infection (Witt et al., 1990).

Denture disinfection has been recommended as an essential procedure for preventing crosscontamination and the maintenance of a healthy oral mucosa. The use of microwave 
irradiation to disinfect dentures and reliners has been suggested (Burns et al., 1990; Polyzois et al., 1995; Webb et al., 1998; Baysan et al., 1998; Thomas et al., 1995; Baysan et al., 1998; Webb et al., 1998) and stimulated as a disinfection model (Fitzpatrick et al., 1978; Lamb et al., 1983; Rohler et al., 1985; Jeng et al., 1987; Friedrich et al., 1988; Najdovski et al., 1991; Arikan et al., 1995; Atmaca et al., 1996; Lin et al., 1999; Kedjarune et al., 1999; Yeo et al., 1999; Nikawa et al., 2000; Jin et al., 2003; Pavarina et al., 2003; Gonçalves et al., 2006; Setlow, 2006; Gonçalves et al., 2007). Since that, researches have been developed in order to ensure the safe use of microwaving disinfection (Table 5).

\begin{tabular}{|c|c|c|c|}
\hline $\begin{array}{c}\text { Authors and } \\
\text { date }\end{array}$ & Materials and groups & $\begin{array}{l}\text { Methods and } \\
\text { measurements }\end{array}$ & $\begin{array}{l}\text { Comments and } \\
\text { conclusions }\end{array}$ \\
\hline $\begin{array}{l}\text { Sanita et al. } \\
(2008)\end{array}$ & $\begin{array}{l}\text { Dentures incubation } \\
\left(37{ }^{\circ} \mathrm{C} / 48 \mathrm{~h}\right) \text { : } \\
\text { (C.albicans, } \\
\text { C.dubliniensis, C. } \\
\text { krusei,C. glabrata and } \\
\text { C.tropicalis). Dentures } \\
\text { microwaved } \\
\text { (650 W/3 min). }\end{array}$ & $\begin{array}{l}\text { Microbial growth on the } \\
\text { plates. }\end{array}$ & $\begin{array}{l}\text { Microwave irradiation } \\
\text { for } 3 \text { min at } 650 \mathrm{~W} \\
\text { resulted in sterilisation } \\
\text { of all complete } \\
\text { dentures. }\end{array}$ \\
\hline $\begin{array}{l}\text { Machado et al. } \\
\text { (2009) }\end{array}$ & $\begin{array}{l}2 \text { hard chairside reline } \\
\text { resins (Kooliner, } \\
\text { DuraLiner II); } 1 \text { heat- } \\
\text { polymerizing denture } \\
\text { base resin (Lucitone } \\
550 \text { ). } \\
\text { Microwave and } \\
\text { chemical disinfection } \\
\text { of samples. }\end{array}$ & $\begin{array}{l}\text { Vickers hardness. } \\
\text { Roughness measurements - } \\
\text { profilometer (diamond } \\
\text { stylus tip radius of } 2 \mu \mathrm{m} \text { ). }\end{array}$ & $\begin{array}{l}\text { Disinfection by } \\
\text { microwave irradiation } \\
\text { did not adversely affect } \\
\text { the hardness of all } \\
\text { materials evaluated. } \\
\text { Roughness varied } \\
\text { among materials and } \\
\text { the effect seems to be } \\
\text { material dependent. }\end{array}$ \\
\hline $\begin{array}{l}\text { Novais et al. } \\
\text { (2009) }\end{array}$ & $\begin{array}{l}\text { Auto polymerised } \\
\text { denture reline } \\
\text { materials (Kooliner). } \\
\text { Conventional heat } \\
\text { polymerized denture } \\
\text { base resin (Lucitone } \\
550 \text { ). }\end{array}$ & $\begin{array}{l}\text { Porosity - after } \\
\text { polymerization; after two } \\
\text { cycles of microwave } \\
\text { disinfection; after seven } \\
\text { cycles of microwave } \\
\text { disinfection; after } 7 \text { days } \\
\text { storage in water at } 37^{\circ} \mathrm{C} \text {. } \\
\text { Number of pores - Scanning } \\
\text { electron microscopy at } \\
\text { magnification } \times 100 \text {. }\end{array}$ & $\begin{array}{l}\text { Differences in the } \\
\text { porosity amongst the } \\
\text { materials and for } \\
\text { different experimental } \\
\text { conditions were } \\
\text { observed following } \\
\text { microwave disinfection. }\end{array}$ \\
\hline $\begin{array}{l}\text { Paranhos et al. } \\
\text { (2009) }\end{array}$ & $\begin{array}{l}\text { Microwave } \\
\text { polymerized acrylic } \\
\text { (Onda-Cryl). } \\
\text { Immersed in } 0.5 \% \\
\mathrm{NaOCl} \text {, and } 1 \% \\
\mathrm{NaOCl} \text {. }\end{array}$ & $\begin{array}{l}\text { Color stability - portable } \\
\text { colorimeter. } \\
\text { Surface roughness - Surftest } \\
\text { SJ-201P surface analyzer } \\
\text { (resolution of } 0.01 \mu \mathrm{m}) \text {. } \\
\text { Flexural strength - universal } \\
\text { testing machine ( } 50 \mathrm{kgf} \\
\text { load cell / crosshead speed } \\
\text { of } 1 \mathrm{~mm} / \mathrm{min}) .\end{array}$ & $\begin{array}{l}\text { Microwave showed } \\
\text { similar results after } \\
\text { treatment in all groups. }\end{array}$ \\
\hline $\begin{array}{l}\text { Dovigo et al. } \\
\text { (2009) }\end{array}$ & $\begin{array}{l}70 \text { water bath } \\
\text { polymerized complete } \\
\text { dentures. }\end{array}$ & $\begin{array}{l}\text { Cultures were interpreted } \\
\text { as positive or negative } \\
\text { growth after disinfection }\end{array}$ & $\begin{array}{l}\text { Microwave irradiation } \\
\text { for } 3 \text { minutes at } 650 \mathrm{~W} \\
\text { produced sterilization }\end{array}$ \\
\hline
\end{tabular}




\begin{tabular}{|c|c|c|c|}
\hline $\begin{array}{c}\text { Authors and } \\
\text { date }\end{array}$ & Materials and groups & $\begin{array}{l}\text { Methods and } \\
\text { measurements }\end{array}$ & $\begin{array}{l}\text { Comments and } \\
\text { conclusions }\end{array}$ \\
\hline & $\begin{array}{l}\text { Inoculated - } \\
\text { Staphylococcus aureus, } \\
\text { Pseudomona } \\
\text { aeruginosa, Bacillus } \\
\text { subtilis, and incubated } \\
\text { for } 24 \text { hours at } 37 \circ \mathrm{C} \text {. } \\
\text { Microwave irradiation } \\
\text { at } 650 \mathrm{~W} \text { for } 3 \\
\text { minutes. }\end{array}$ & treatment. & $\begin{array}{l}\text { of complete dentures } \\
\text { contaminated with } S \text {. } \\
\text { aureus and } P \text {. aeruginosa. } \\
\text { Dentures contaminated } \\
\text { with } B \text {. subtilis can be } \\
\text { disinfected by } \\
\text { microwave irradiation } \\
\text { after } 3 \text { and } 5 \text { minutes at } \\
650 \mathrm{~W} \text {. }\end{array}$ \\
\hline $\begin{array}{l}\text { Montagner et } \\
\text { al. (2009) }\end{array}$ & $\begin{array}{l}\text { Microwave-cured } \\
\text { acrylic resin } \\
\text { specimens } \\
\text { contaminated with } \\
\text { Candida albicans } \\
\text { antifungal action of } \\
\text { different disinfection } \\
\text { agents ( } 2 \% \\
\text { chlorhexidine solution } \\
\text { / } 10 \text { min; } 0.5 \% \\
\text { sodium hypochlorite } \\
\text { / } 10 \text { min; modified } \\
\text { sodium hypochlorite } \\
\text { / } 10 \text { min; effervescent } \\
\text { agent / } 5 \text { min; } \\
\text { hydrogen peroxide } 10 \\
\text { v / } 30 \text { min). }\end{array}$ & $\begin{array}{l}\text { Culture media turbidity - } \\
\text { spectrophotometrically } \\
\text { according to the } \\
\text { transmittance degree (the } \\
\text { higher the transmittance the } \\
\text { stronger the antimicrobial } \\
\text { action). }\end{array}$ & $\begin{array}{l}\text { Sodium } \\
\text { hypochloritebased } \\
\text { substances and } \\
\text { hydrogen peroxide are } \\
\text { more efficient } \\
\text { disinfectants against } C \text {. } \\
\text { albicans than } 2 \% \\
\text { chlorhexidine solution } \\
\text { and the effervescent } \\
\text { agent. }\end{array}$ \\
\hline $\begin{array}{l}\text { Sartori et al. } \\
(2008)\end{array}$ & $\begin{array}{l}\text { Denture base resin } \\
\text { disinfection } \\
\text { procedures performed } \\
\text { twice: Chemical } \\
\text { disinfection } \\
\text { (immersion in } \\
\text { 100ppm chloride } \\
\text { solution for } 24 \mathrm{~h} \text { ), and } \\
\text { Microwave } \\
\text { Disinfection } \\
\text { (irradiation } \\
\text { at } 690 \mathrm{~W} \text { ). }\end{array}$ & $\begin{array}{l}\text { Knoop microhardness } \\
\text { dimensional stability - } \\
\text { weighing a vinyl } \\
\text { polysiloxane film } \\
\text { reproducing the gap } \\
\text { between resin base and } \\
\text { master model. }\end{array}$ & $\begin{array}{l}\text { Knoop microhardness } \\
\text { was not modified by } \\
\text { any disinfection } \\
\text { procedure, but } \\
\text { decreased over time. } \\
\text { Microwaved denture } \\
\text { resin bases had gradual } \\
\text { increase of distortion } \\
\text { over time. }\end{array}$ \\
\hline $\begin{array}{l}\text { Mima et al. } \\
(2008)\end{array}$ & $\begin{array}{l}\text { Hard chairside reline } \\
\text { resin samples } \\
\text { inoculation by } \\
\text { Pseudomonas } \\
\text { aeruginosa, } \\
\text { Staphylococcus aureus, } \\
\text { Candida albicans, and } \\
\text { Bacillus subtilis. } \\
\text { Samples microwaved } \\
\text { at } 650 \text { W for } 1,2,3,4, \\
\text { or } 5 \text { minutes before } \\
\text { serial dilutions and } \\
\text { platings. }\end{array}$ & $\begin{array}{l}\text { Colonies on plates were } \\
\text { counted after } 48 \text { hours of } \\
\text { Incubation. }\end{array}$ & $\begin{array}{l}3 \text { minutes of microwave } \\
\text { irradiation can be used } \\
\text { for acrylic resin } \\
\text { sterilization. }\end{array}$ \\
\hline
\end{tabular}




\begin{tabular}{|c|c|c|c|}
\hline $\begin{array}{l}\text { Authors and } \\
\text { date }\end{array}$ & Materials and groups & $\begin{array}{l}\text { Methods and } \\
\text { measurements }\end{array}$ & $\begin{array}{c}\text { Comments and } \\
\text { conclusions }\end{array}$ \\
\hline $\begin{array}{l}\text { Ribeiro et al. } \\
(2008)\end{array}$ & $\begin{array}{l}\text { Three polymethyl } \\
\text { methacrylate (PMMA) } \\
\text { resins: a conventional } \\
\text { water-bath, heat } \\
\text { activated acrylic } \\
\text { resin (Lucitone 550), } \\
\text { rapid polymerizing } \\
\text { acrylic } \\
\text { resin (QC-20-QC)), } \\
\text { and microwave } \\
\text { activated acrylic resin } \\
\text { (Acron MC-AC). } \\
\text { Two cycles of } \\
\text { microwave } \\
\text { disinfection (650W for } \\
6 \text { min - once, twice } \\
\text { and seven times). }\end{array}$ & $\begin{array}{l}\text { Shear bond strength } \\
\text { between denture teeth and } \\
\text { acrylic resins having } \\
\text { different polymerization } \\
\text { cycles - knife-edge shear } \\
\text { test in a universal test } \\
\text { machine (MTS-810). }\end{array}$ & $\begin{array}{l}\text { The shear bond strength } \\
\text { between the denture } \\
\text { teeth and the acrylic } \\
\text { resins Acron MC and } \\
\text { Lucitone } 550 \text { was not } \\
\text { affected by microwave } \\
\text { disinfection. } \\
\text { After two cycles of } \\
\text { microwave disinfection, } \\
\text { the shear bond strength } \\
\text { of teeth to QC- } 20 \text { acrylic } \\
\text { resin was increased. } \\
\text { Seven cycles of } \\
\text { microwave disinfection } \\
\text { significantly decreased } \\
\text { the shear bond strength } \\
\text { between teeth and QC- } \\
20 \text { acrylic resin. }\end{array}$ \\
\hline $\begin{array}{l}\text { Pero et al. } \\
(2007)\end{array}$ & $\begin{array}{l}\text { Two heat-activated } \\
\text { denture base resins - } \\
\text { conventional (Clássico } \\
\text { - water bath); } \\
\text { designed for } \\
\text { microwave } \\
\text { polymerization } \\
\text { (Onda-Cryl - } \\
\text { manufacturing } \\
\text { microwave cycle, } \\
\text { short microwave cycle } \\
\text { and long microwave } \\
\text { cycle). } \\
\text { Thicknesses - 2.0, 3.5, } \\
\text { and } 5.0 \text { mm. } \\
\text { Immersion in water. }\end{array}$ & Porosity. & $\begin{array}{l}\text { Microwave } \\
\text { polymerization cycles } \\
\text { and the specimen } \\
\text { thickness of acrylic } \\
\text { resin influenced } \\
\text { porosity. } \\
\text { Porosity differences } \\
\text { were not observed in } \\
\text { the polymerized resin } \\
\text { bases in the water bath } \\
\text { cycle for any thickness. }\end{array}$ \\
\hline $\begin{array}{l}\text { Consani et al. } \\
\text { (2007) }\end{array}$ & $\begin{array}{l}\text { Maxillary denture } \\
\text { base adaptation } \\
\text { (Clássico - } 74^{\circ} \\
\text { C for } 9 \text { hours). } \\
2 \text { different flasks: } \\
\text { closure methods } \\
\text { (traditional flask } \\
\text { closure and } \\
\text { Restriction System } \\
\text { flask closure } \\
\text { methods). } \\
\text { Simulated disinfection } \\
\text { in } 150 \text { mL distilled } \\
\text { water in a microwave } \\
\text { oven at } 650 \mathrm{~W} \text { for } 3 \\
\text { minutes. }\end{array}$ & $\begin{array}{l}3 \text { transverse cuts on denture } \\
\text { bases: distal of canines, } \\
\text { mesial of first molars, and } \\
\text { posterior palatal region. } \\
\text { Measurements of } \\
\text { desadaptation: optical } \\
\text { micrometer at } 5 \text { points. }\end{array}$ & $\begin{array}{l}\text { Simulated disinfection } \\
\text { by microwave energy } \\
\text { improved denture base } \\
\text { adaptation. }\end{array}$ \\
\hline
\end{tabular}




\begin{tabular}{|c|c|c|c|}
\hline $\begin{array}{c}\text { Authors and } \\
\text { date }\end{array}$ & Materials and groups & $\begin{array}{c}\text { Methods and } \\
\text { measurements }\end{array}$ & $\begin{array}{c}\text { Comments and } \\
\text { conclusions }\end{array}$ \\
\hline Seo et al. (2007) & $\begin{array}{l}\text { Lucitone } 550 \text { denture } \\
\text { bases ( } 2 \text { - and } 4 \text { - } \\
\text { mmthick). } \\
\text { Intact and } \\
\text { autopolymerizing } \\
\text { resin relined. } \\
1 \text { cycle of microwave } \\
\text { disinfection } \\
\text { (650W/6min); daily } \\
\text { microwave } \\
\text { disinfection for } 7 \\
\text { days. }\end{array}$ & $\begin{array}{l}\text { Dimensional stability - area } \\
\text { between } 5 \text { removable pins } \\
\text { on the standard brass cast } \\
\text { measured with a Nikon } \\
\text { optical comparator, before } \\
\text { and after treatment. }\end{array}$ & $\begin{array}{l}\text { Microwave disinfection } \\
\text { produced increased } \\
\text { shrinkage of intact } \\
\text { specimens and those } \\
\text { relined. }\end{array}$ \\
\hline $\begin{array}{l}\text { Pavan et al. } \\
(2007)\end{array}$ & $\begin{array}{l}\text { Four soft lining } \\
\text { materials (Molloplast- } \\
\text { B, Ufi Gel P, Eversoft, } \\
\text { Mucopren soft). } \\
\text { Microwave oven for } 3 \\
\text { minutes at } 500 \mathrm{~W} \text {. } \\
\text { Disinfectant solutions } \\
\text { for } 10 \text { minutes. }\end{array}$ & $\begin{array}{l}\text { Hardness - Shore A } \\
\text { durometer. }\end{array}$ & $\begin{array}{l}\text { Application disinfection } \\
\text { cycles did not change } \\
\text { the Shore A hardness } \\
\text { values for the materials. }\end{array}$ \\
\hline $\begin{array}{l}\text { Sartori et al. } \\
(2006)\end{array}$ & $\begin{array}{l}\text { Denture resin bases. } \\
\text { Chemical disinfection. } \\
\text { Microwave } \\
\text { disinfection. } \\
\text { Twice with a 7-day } \\
\text { interval between them. }\end{array}$ & $\begin{array}{l}\text { Internal adaptation: } \\
\text { weighing a vinyl } \\
\text { polysiloxane film } \\
\text { reproducing the gap } \\
\text { between the resin base and } \\
\text { the master model. }\end{array}$ & $\begin{array}{l}\text { Microwave disinfection } \\
\text { had gradual increase of } \\
\text { misfit bases immersed } \\
\text { in chloride solution did } \\
\text { not differ from the } \\
\text { control group. }\end{array}$ \\
\hline $\begin{array}{l}\text { Silva et al. } \\
(2006)\end{array}$ & $\begin{array}{l}\text { Complete resin } \\
\text { dentures. } \\
\text { Inoculated by Candida } \\
\text { albicans,Streptoccus } \\
\text { aureus, Bacillus subtilis, } \\
\text { and Pseudomonas } \\
\text { Aeruginosa. } \\
\text { Disinfection by } \\
\text { microwave irradiation } \\
\text { at } 650 \mathrm{~W} \text { for } 6 \text { minutes. }\end{array}$ & $\begin{array}{l}\text { Effectiveness of microwave } \\
\text { disinfection: count of the } \\
\text { number of colony-forming } \\
\text { units. }\end{array}$ & $\begin{array}{l}\text { Microwave irradiation } \\
\text { for } 6 \text { minutes at } 650 \mathrm{~W} \\
\text { produced sterilization } \\
\text { and disinfection of } \\
\text { complete dentures. }\end{array}$ \\
\hline $\begin{array}{l}\text { Moura et al. } \\
(2006)\end{array}$ & $\begin{array}{l}\text { Classico (water bath - } \\
\left.9 \text { h at } 74^{\circ} \mathrm{C}\right) \text {. } \\
\text { Onda Cryl } \\
\text { (Microwave energy } 3 \\
\text { min at } 360 \mathrm{~W}+4 \mathrm{~min} \\
\text { pause }+3 \mathrm{~min} \text { at } 810 \\
\mathrm{~W}) . \\
\text { Infection - (Candida } \\
\text { albicans, Candida } \\
\text { tropicalis,Candida } \\
\text { dubliniensis, Candida } \\
\text { glabrata). }\end{array}$ & $\begin{array}{l}\text { Roughness - profilometer. } \\
\text { Surface free energy - } \\
\text { contact angle of a sessile } \\
\text { drop of water. }\end{array}$ & $\begin{array}{l}\text { The polymerization } \\
\text { method, heat versus } \\
\text { microwave, did not } \\
\text { influence Candida species } \\
\text { adherence values. } \\
\text { There is no correlation } \\
\text { regarding surface free } \\
\text { energy, surface } \\
\text { roughness and the } \\
\text { adhesion of Candida } \\
\text { species. } \\
\text { Heat polymerized } \\
\text { acrylic resin showed } \\
\text { highest surface free } \\
\text { energy values. }\end{array}$ \\
\hline
\end{tabular}




\begin{tabular}{|c|c|c|c|}
\hline $\begin{array}{c}\text { Authors and } \\
\text { date }\end{array}$ & Materials and groups & $\begin{array}{l}\text { Methods and } \\
\text { measurements }\end{array}$ & $\begin{array}{l}\text { Comments and } \\
\text { conclusions }\end{array}$ \\
\hline $\begin{array}{l}\text { Machado et al. } \\
\text { (2006) }\end{array}$ & $\begin{array}{l}\text { Cylindrical }(30 \times 3.9 \\
\text { mm) resin specimens } \\
(\text { Luciitone } 199) . \\
\text { Reline materials } \\
\text { packed in resin. } \\
\text { Twice microwave } \\
\text { irradiation ( } 650 \mathrm{~W} \text { for } \\
6 \text { minutes). }\end{array}$ & $\begin{array}{l}\text { Torsional test }(0.1 \\
\text { Nm/min). } \\
\text { Torsional strengths }(\mathrm{MPa}) \text {. } \\
\text { Mode of failure. }\end{array}$ & $\begin{array}{l}\text { Microwave disinfection } \\
\text { cycles do not decrease } \\
\text { the torsional bond } \\
\text { strengths between the } \\
\text { hard reline resins. } \\
\text { Disinfection cycles on } \\
\text { reline material may be } \\
\text { clinically significant and } \\
\text { requires further study. }\end{array}$ \\
\hline $\begin{array}{l}\text { Machado et al. } \\
\text { (2005) }\end{array}$ & $\begin{array}{l}\text { Acrylic resin } \\
\text { (Lucitone 199). } \\
\text { Resilient lining } \\
\text { materials (GC Reline } \\
\text { Extra Soft and } \\
\text { Dentusil). } \\
\text { Samples irradiated } \\
\text { twice, with } 650 \mathrm{~W} / 6 \\
\text { min; samples } \\
\text { irradiated daily for } 7 \\
\text { total cycles. }\end{array}$ & $\begin{array}{l}\text { To peel the resilient } \\
\text { adhesive - screw tensile } \\
\text { tester (Sintec } 2 / G \text { ) (visual } \\
\text { analyzed of resin interface). } \\
\text { Hadness - Shore A } \\
\text { durometer (before and after } \\
\text { treatment). }\end{array}$ & $\begin{array}{l}\text { Microwave disinfection } \\
\text { did not compromise the } \\
\text { hardness of either } \\
\text { resilient liners or their } \\
\text { adhesion to the denture } \\
\text { base resin Lucitone } 199 \text {. } \\
\text { The hardness of the } \\
\text { Lucitone } 550 \text { denture } \\
\text { base resin specimens } \\
\text { was not affected by } \\
\text { either disinfection } \\
\text { method evaluated. }\end{array}$ \\
\hline $\begin{array}{l}\text { Campanha et } \\
\text { al. (2005) }\end{array}$ & $\begin{array}{l}6 \text { brands of artificial } \\
\text { teeth. } \\
\text { Microwave } \\
\text { sterilization at } 650 \mathrm{~W} \\
\text { for } 6 \text { minutes. }\end{array}$ & $\begin{array}{l}\text { Hardness - Vickers } \\
\text { diamond indentator. }\end{array}$ & $\begin{array}{l}\text { Two cycles of } \\
\text { microwave sterilization } \\
\text { did not affect the } \\
\text { hardness of most of the } \\
\text { acrylic resin denture } \\
\text { teeth tested. } \\
\text { Microwave sterilization } \\
\text { significantly decreased } \\
\text { the hardness of acrylic } \\
\text { resin artificial teeth. }\end{array}$ \\
\hline $\begin{array}{l}\text { Pavarina et al. } \\
\text { (2005) }\end{array}$ & $\begin{array}{l}\text { Denture base resin } \\
\text { (Lucitone 550). } \\
\text { Five hard chairside } \\
\text { reline resins } \\
\text { (Kooliner, Duraliner } \\
\text { II, Tokuso Rebase } \\
\text { Fast, Ufi Gel Hard, } \\
\text { and New Truliner). } \\
\text { Microwave } \\
\text { disinfection (650W/6 } \\
\text { min): two cycles and } \\
\text { seven cycles. }\end{array}$ & $\begin{array}{l}\text { Flexural strength }(\mathrm{MPa})-3 \\
\text { point bend fixture in a MTS } \\
\text { machine and loaded until } \\
\text { failure. }\end{array}$ & $\begin{array}{l}\text { Microwave disinfection } \\
\text { do not adversely affect } \\
\text { the flexural strength of } \\
\text { all tested materials. }\end{array}$ \\
\hline $\begin{array}{l}\text { Rodrigues } \\
\text { Garcia et al. } \\
\text { (2004) }\end{array}$ & $\begin{array}{l}\text { Microwave-cured } \\
\text { acrylic resin disc } \\
\text { shaped samples. } \\
3 \text { cleansing treatments } \\
\text { (polident, } \\
\text { manipulation } \\
\text { pharmacy cleanser, } \\
\text { and water). }\end{array}$ & $\begin{array}{l}\text { Hardness. } \\
\text { Roughness. }\end{array}$ & $\begin{array}{l}\text { Manipulated cleanser } \\
\text { containing sodium } \\
\text { perborate increased } \\
\text { surface roughness and } \\
\text { hardness. }\end{array}$ \\
\hline
\end{tabular}




\begin{tabular}{|c|c|c|c|}
\hline $\begin{array}{c}\text { Authors and } \\
\text { date }\end{array}$ & Materials and groups & $\begin{array}{c}\text { Methods and } \\
\text { measurements }\end{array}$ & $\begin{array}{c}\text { Comments and } \\
\text { conclusions }\end{array}$ \\
\hline Seo et al. (2007) & $\begin{array}{l}\text { 2- and 4-mm-thick } \\
\text { denture bases } \\
\text { (Lucitone 550). } \\
\text { Reline with } 2 \text { mm of } \\
\text { autopolymerizing } \\
\text { resin (Tokuso Rebase } \\
\text { Fast, Ufi Gel Hard, } \\
\text { Kooliner, or New } \\
\text { Truliner). }\end{array}$ & $\begin{array}{l}\text { Dimensional stability - } 5 \\
\text { removable pins on the } \\
\text { standard brass cast - area } \\
\text { (mm) formed by the } \\
\text { distance between } 5 \text { pins } \\
\text { (Nikon optical comparator). }\end{array}$ & $\begin{array}{l}\text { Microwave disinfection } \\
\text { produced increased } \\
\text { shrinkage of intact } \\
\text { specimens and those } \\
\text { relined with New } \\
\text { Truliner and Kooliner. }\end{array}$ \\
\hline $\begin{array}{l}\text { Neppelenbroek } \\
\text { et al. (2003) }\end{array}$ & $\begin{array}{l}\text { Treatment of } 15 \\
\text { denture patients with } \\
\text { Candida- related } \\
\text { denture stomatitis. } \\
\text { Upper denture } \\
\text { microwaved ( } 650 \mathrm{~W} / 6 \\
\text { min) three times per } \\
\text { week for } 30 \text { days; } \\
\text { conjunction with } \\
\text { topical application of } \\
\text { miconazole three } \\
\text { times per day for } 30 \\
\text { days; antifungal } \\
\text { therapy only. }\end{array}$ & $\begin{array}{l}\text { Cytological smears and } \\
\text { mycological cultures - after } \\
\text { and before treatment (days } \\
15 \text { and } 30 \text { follow-up). }\end{array}$ & $\begin{array}{l}\text { Microwaving dentures } \\
\text { was effective for the } \\
\text { treatment of denture } \\
\text { stomatitis. }\end{array}$ \\
\hline $\begin{array}{l}\text { Banting et al. } \\
(2001)\end{array}$ & $\begin{array}{l}\text { Thirty-four subjects } \\
\text { with a positive test for } \\
\text { C. albicans } \\
\text { pseudohyphae. } \\
\text { Subjects in the } \\
\text { microwave treatment } \\
\text { group - maxillary } \\
\text { denture microwaved } \\
\text { ( } 850 W / 1 \mathrm{~m}) \text {. } \\
\text { Procedure repeated } \\
\text { three times. } \\
\text { Standard denture } \\
\text { soak treatment - } \\
\text { liquid disinfect the } \\
\text { dentures (.2\% } \\
\text { chlorhexidine } \\
\text { digluconate solution } \\
\text { overnight for } 14 \text { days). }\end{array}$ & $\begin{array}{l}\text { Infestation of the tissue } \\
\text { surface of the maxillary } \\
\text { denture; cytological smears. }\end{array}$ & $\begin{array}{l}\text { Patients whose dentures } \\
\text { were microwaved have } \\
\text { delayed dramatically } \\
\text { reinfestation of the } \\
\text { denture surface and } \\
\text { infection of the adjacent } \\
\text { soft tissue. } \\
\text { Microwave treatment is } \\
\text { not recommended for } \\
\text { all dentures and should } \\
\text { be used with caution. }\end{array}$ \\
\hline $\begin{array}{l}\text { Dixon et al. } \\
\text { (1999) }\end{array}$ & $\begin{array}{l}\text { Denture base soft } \\
\text { liners and } \\
\text { heatpolymerized } \\
\text { Acrylic resin denture } \\
\text { base material. } \\
\text { Inoculation with C. } \\
\text { albicans. } \\
\text { Irradiation in a } 60 \mathrm{~Hz} \\
\text { microwave oven for }\end{array}$ & $\begin{array}{l}\text { Efficacy of microwave } \\
\text { irradiation against } C \text {. } \\
\text { albicans. } \\
\text { Effect of irradiation on the } \\
\text { materials hardness. } \\
\text { C. Albicans growth assessed } \\
\text { with streaked blood agar } \\
\text { plates and thioglycollate } \\
\text { broth. }\end{array}$ & $\begin{array}{l}\text { Five-minute irradiation, } \\
\text { while immersed in } \\
\text { water, killed all } C \text {. } \\
\text { albicans present on the } \\
\text { materials tested; } \\
\text { repeated 5-minute } \\
\text { irradiation significantly } \\
\text { affected the hardness of } \\
\text { only the PermaSoft. }\end{array}$ \\
\hline
\end{tabular}




\begin{tabular}{|c|l|l|l|}
\hline $\begin{array}{c}\text { Authors and } \\
\text { date }\end{array}$ & Materials and groups & $\begin{array}{c}\text { Methods and } \\
\text { measurements }\end{array}$ & $\begin{array}{c}\text { Comments and } \\
\text { conclusions }\end{array}$ \\
\hline & $\begin{array}{l}\text { 5minutes (10 - and 15- } \\
\text { minute irradiation; } \\
\text { repeated 5-minute } \\
\text { irradiation cycles). }\end{array}$ & Shore A hardness material. & \\
\hline
\end{tabular}

Table 5. Studies about the effects of microwave disinfection

\section{Conclusion}

The use of microwave energy in the processing of acrylic resin is based on both, classic and recent studies. The observed differences when using microwave or water-bath curing usually are not clinically significant where mechanical properties of microwave and waterbath cured resins are not significant in the resins properties. The frequency and size of porosity in thick specimens could be reduced to $30 \%$ by a longer polymerization time at a lower wattage. Microwave curing as a rule, has little effect on the properties of resins when the choice of a suitable power and polymerization time are adequate, reducing porosity or dimensional alteration to a minimum level.

Because it offers some important physical properties as good as conventional processing, along with the advantage of being a quicker and easier method, it should also be considered in processing removable partial dentures or complete dentures, and as a disinfection method of resin prosthesis.

\section{References}

Al Doori, D.; Huggett, R. \& Bates, J.F. (1988). A comparison of denture base acrylic resins polymerized by microwave irradiation and by conventional water bath curing systems. Dental Materials, Vol.4, No.1, 25-32, ISSN 0109-5641

Al-Hanbali, E.; Kalleway, J.P. \& Howlett, J.A. (1991). Acrylic denture distortion following double processing with microwave or heat. Journal of Dentistry, Vol.91, No.3, (Jun) 176-180, ISSN 0300-5712

Alkhatib, M.B.; Goodacre, C.J.; Swartz, M.L.; Munoz-Viveros, C.A. \& Andres, C.J. (1990). Comparison of microwave-polymerized denture base resins. The International Journal of Prosthodontics, Vol.3, No.3, (May-Jun) 249-255, ISSN 0893-2174

Al-Mulla, M.A.S.; Huggett, R.; Brooks, S.C. \& Murphyt, W.M. (1988). Some physical and mechanical properties of a visible light-activated material. Dental Materials, Vol.4, No.4, (Aug), 197-200, ISSN 0109-5641

Arikan, A.; Kulak, Y. \& Kadir, T. (1995). Comparison of different treatment methods for localized and generalized simple denture stomatitis. Journal of Oral Rehabilitation, Vol.22, No.5, (May) 365-369, ISSN 0305-182X

Assunção, W.G.; Barão, V.A.R.; Pita, M.S. \& Goiato, M.C. (2009). Effect of polymerization methods and thermal cycling on color stability of acrylic resin denture teeth. Journal of Prosthetic Dentistry, Vol.102, No.6, (Dec) 385-392, ISSN 0022-3913

Atmaca, S.; Akdag, Z.; Dasdag, S. \& Celik, S. (1996). Effect of microwaves on survival of some bacterial strains. Acta microbiologica et immunologica Hungarica, Vol.43, No.4, 371-378, ISSN 1217-8950 
Austin, A.T. \& Basker, R.M. (1982). Residual monomer levels in denture bases. The effects of varying short curing cycles. British Dental Journal, Vol.153, No.12, (Dec) 424-426, ISSN 0007-0610

Austin, A.T. \& Basker, R.M. (1980). The level of residual monomer in acrylic denture base materials. British Dental Journal, Vol.149, No.10, (Nov) 281-286, ISSN 0007-0610

Azzarri, M.J.; Cortizo, M.S. \& Alessandrini, J.L. (2003). Effect of the curing conditions on the properties of an acrylic denture base resin microwave-polymerised. Journal of Dentistry, Vol.31, 463-468, ISSN 0300-5712

Bafile, M.; Graser, G.N.; Myers, M.L. \& Li, E.K. (1991). Porosity in denture resin cured by microwave energy. Journal of Prosthetic Dentistry, Vol.66, 269-274, ISSN 0022-3913

Banting, D.W. \& Hill, S.A. (2001). Microwave disinfection of dentures for the treatment of oral candidiasis. Special Care in Dentistry, Vol.21, No.1, 4-8, ISSN 0275-1879

Barbosa, D.B.; Compagnoni, M.A. \& Leles, C.R. (2002). Changes in occlusal vertical dimension in microwave processing of complete dentures. Brazilian Dental Journal, Vol.13, No.3, 197-200, ISSN 0103-6440

Bartoloni, J.A.; Murchison, D.F.; Wofford, D.t. \& Sarkar, N.K. (2000). Degree of conversion in denture base materials for varied polymerization techniques. Journal of OralRehabilitation, Vol.27, 488-493, ISSN 0305-182X

Baysan, A.; Whitley, R. \& Wright, P.S. (1998). Use of microwave energy to disinfect a longterm soft lining material contaminated with Candida albicans or Staphylococcus aureus. Journal of Prosthetic Dentistry, Vol.79, 454-458, ISSN 0022-3913

Blagojevic, V. \& Murphy, V.M. (1999). Microwave polymerization of denture base materials. A comparative study. Journal of Oral Rehabilitation, Vol.26, 804-808, ISSN 0305-182X

Botega, D.M.; Machado, T.S.; Mello, J.A.N.; Rodrigues Garcia, R.C.M. \& Del Bel Cury, A.A. (2004). Polymerization time for a microwavecured acrylic resin with multiple flasks. Brazilian Oral Research, Vol.18, No.1, 23-28, ISSN 1806-8324

Braun, K.O.; Rodrigues Garcia, R.C.M.; Rizzatti-Barbosa, C.M. \& Del Bel Cury, A.A. (2000). Linear dimensional change of denture base resins cured by microwave activation. Brazilian Oral Research, Vol.14, No.3, 278-282, ISSN 1806-8324

Braun, K.O.; Del Bel Cury, A.A. \& Cury J.A. (1988). Use of microwave energy for processing acrylic near metal. Brazilian Oral Research, Vol.12, No.2, 173-180, ISSN 1806-8324

Brosky, M.E.; Pesun, I.J.; Morrison, B.; Hodges, J.S.; Lai, J.H. \& Liljemark, W. (2003). Clinical evaluation of resilient denture liners. Part 2: Candida count and speciation. Journal of Prosthodontics, Vol.12, 162-167, ISSN 1059-941X

Bulad, K.; Taylor, R.L.; Verran, J. \& McCord, J.F. (2004). Colonization and penetration of denture soft lining materials by Candida albicans. Dental Materials, Vol.20, 167-175, ISSN 0109-5641

Burns, D.R.; Kazanoglu, A.; Moon, P.C. \& Gunsolley, J.C. (1990). Dimensional stability of acrylic resin materials after microwave sterilization. International Journal of Prosthodontics, Vol.3, 489-493, ISSN 0893-2174

Campanha, N.H.; Pavarina, A.C.; Vergani, C.E. \& Machado, A.L. (2005). Effect of microwave sterilization and water storage on the Vickers hardness of acrylic resin denture teeth. Journal of Prosthetic Dentistry, Vol.93, 483-487, ISSN 0022-3913 
Caycik, S. \& Jagger, R.G. (1992). The effect of cross-linking chain length on mechanical properties of a dough-molded poly(methylmethacrylate) resin. Dental Materials, Vol.8, 153-157, ISSN 0109-5641

Chen, M.; Siochi, E.J.; Ward, T.C. \& McGrath, J.E. (1993). Basic ideas of microwave processing of polymers. Polymer Engineering $\mathcal{E}$ Science, Vol.33, 1092-1109, ISSN 0032-3888

Chen, J.C.; Lacefield, W.R. \& Castleberry, D.J. (1988) Effect of denture thickness and curing cycle on the dimensional stability of acrylic resin denture bases. Dental Materials, Vol.4, No.1, (Feb) 20-24, ISSN 0109-5641

Compagnoni, M.A.; Barbosa, D.B.; de Souza, R.F. \& Pero, A.C. (2004). The effect of polymerization cycles on porosity of microwave-processed denture base resin. Journal of Prosthetic Dentistry, Vol.91, 281-285, ISSN 0022-3913

Consani, R.L.X.; Mesquita, M.F.; Nobilo, M.A.A. \& Henriques, G.E. (2007). Influence of simulated microwave disinfection on complete denture base adaptation using different flask closure methods. Journal of Prosthetic Dentistry, Vol.97, 173-178, ISSN 0022-3913

Craig, R.G. (1993). Restorative Dental Materials, 9th ed., pp. 514-530, Mosby, ISBN 0801668727, Saint Louis.

Davenport, J.C. (1970. The oral distribution of candida in denture stomatitis. British Dental Journal, Vol.129, 151-156, ISSN 0007-0610

De Clerck, J.P. (1987). Microwave polymerization of acrylic resins used in dental prostheses. Journal of Prosthetic Dentistry, Vol.57, 650-658, ISSN 0022-3913

Del Bel Cury, A.A.; Rached, R.N. \& Ganzarolli, S.M. (2001). Microwave-cured acrylic resins and silicone-gypsum moulding technique. Journal of Oral Rehabilitation, Vol.28, 433438, ISSN 0305-182X

Del Bel Cury, A.A.; Rodrigues Jr, A.L. \& Panzeri, H. (1994). Acylic resins polymerized by microwave energy, conventional water bath curing and self-curing system: physical properties. Revista da Faculdade de Odontologia da Universidade de São Paulo, Vol.8, No.4, 243-249, ISSN 0103-0663

Dixon, D.L.; Breeding, L.C. \& Faler, T.A. (1999). Microwave disinfection of denture base materials colonized with Candida albicans. Journal of Prosthetic Dentistry, Vol.81, 207-214, ISSN 0022-3913

Dovigo, L.N.; Pavarina, A.C.; Ribeiro, D.G.; Oliveira, J.A.; Vergani, C.E. \& Machado, A.L. (2009). Microwave Disinfection of Complete Dentures Contaminated In Vitro with Selected Bacteria. Journal of Prosthodontics, Vol.18, 611-617, ISSN 1059-941X

Faot, F.; Rodrigues Garcia, R.C.M. \& Del Bel Cury, A.A. (2008). Fractographic analysis, accuracy of fit and impact strength of acrylic resin. Brazilian Oral Research, Vol.22, No.4, 334-339, ISSN 1806-8324

Faraj, S.A. \& Ellis, B. (1979). The effect of processing temperatures on the exotherm, porosity and properties of acrylic denture base. British Dental Journal, Vol.147, 209-212, ISSN 0007-0610

Fitzpatrick, J.A.; Kwao-Paul, J. \& Massey, J. (1978). Sterilization of bacteria by means of microwave heating. Journal of Clinical Engineering, Vol.3, No.1, 44-47, ISSN 03638855 
Frangou, M.J. \& Polyzois, G.L. (1993). Effect of microwave polymerisation on indentation creep, recovery and hardness of acrylic denture base materials. The European Journal of Prosthodontics and Restorative Dentistry, Vol.1, 111-115, ISSN 0965-7452

Friedrich Jr, E.G. \& Phillips, L.E. (1988). Microwave sterilization of Candida on underwear fabric. A preliminary report. The Journal of Reproductive Medicine, Vol.33, 421-422, ISSN 0024-7758

Ganzarolli, S.M.; Mello, J.A.N.; Shinkai, R.S. \& Del Bel Cury, A.A. (2007). Internal Adaptation and Some Physical Properties of Methacrylate-based Denture Base Resins Polymerized by Different Techniques. Journal of Biomedical Materials Research. Part B, Applied biomaterials, Vol.82B, 169-173, ISSN 1552-4973

Ganzarolli, S.M.; Rached, R.N.; Rodrigues Garcia, R.C. M. \& Del Bel Cury, A.A. (2002). Effect of cooling procedure on final denture base adaptation. Journal of Oral Rehabilitation, Vol.29, 787-790, ISSN 0305-182X

Gonçalves, A.R.; Machado, A.L.; Giampaolo, E.T.; Pavarina, A.C. \& Vergani, C.E. (2006). Linear dimensional changes of denture base and hard chair-side reline resins after disinfection. Journal of Applied Polymer Science, Vol.102, No.2, 1821-1825, ISSN 02719460

Gonçalves, A.R.; Machado, A.L.; Vergani, C.E.; Giampaolo, E.T. \& Pavarina, A.C. (2007). Effect of disinfection on adhesion of reline polymers. Journal of Adhesion, Vol.83, 139-150, ISSN 0021-8464

Gourdinne, A.; Maassarani, A.; Monchaux, P.; Aussudre, S. \& Thourel, L. (1979). Crosslinking of thermosetting resins by microwave heating: quantitative approach. Polymer Preprints, Vol.20, 471-474, ISSN 0032-3934

Harman, I.E. (1949). Effects of time and temperature on polymerization of a methacrylate resin denture base. Journal of the American Dental Association, Vol.38, 188, ISSN 00028177

Hayden, W.J. (1986). Transverse strength of microwave-cured denture baseplates. General Dentistry, Vol.34, 367-371, ISSN 0363-6771

Hogan, P.F. \& Mori, T. (1990). Development of a method of continuous temperature measurement for microwave denture processing. Dental Materials Journal, Vol.9, 111, ISSN 0287-4547

Huggett, R.; Brooks, S.C. \& Bates, J.F. (1984). The effect of different curing cycles on levels of residual monomer in acrylic resin denture base materials. Quintessence of Dental Technology, Vol.8, 365-371, ISSN 0362-0913

Ilbay, S.G.; Guöener, S. \& Alkumru, H.N. (1994). Processing dentures using a microwave technique. Journal of Oral Rehabilitation, Vol.21, 103-109, ISSN 0305-182X

Jacob, J.; Chia, L.H.L. \& Boey, F.Y.C. (1997). Microwave polymerization of poly(methylacrylate): conversion studies at variable power. Journal of Applied Polymer Science, Vol.63, 787-797, ISSN 0271-9460

Jagger, R.G. (1996). Dimensional accuracy of thermoformed polymethyl methacrylate. Journal of Prosthetic Dentistry, Vol.76, No.6, (Dec) 573-575, ISSN 0022-3913

Jeng, D.K.; Kaczmarek, K.A.; Woodworth, A.G. \& Balasky G. (1987). Mechanism of microwave sterilization in the dry state. Applied and Environmental Microbiology, Vol.53, No.9, (Sept) 2133-2137, ISSN 0099-2240

Jerolimov, V.; Brooks, S.C.; Huggett, R. \& Bates, J.F. (1989). Rapid curing of acrylic denturebase materials. Dental Materials, Vol.5, 18-22, ISSN 0109-5641 
Jin, C.; Nikawa, H.; Makihira, S.; Hamada, T.; Furukawa, M. \& Murata, H. (2003). Changes in surface roughness and colour stability of soft denture lining materials caused by denture cleansers. Journal of Oral Rehabilitation, Vol.30, 125-130, ISSN 0305-182X

Kedjarune, U.; Charoenworaluk, N. \& Koontongkaew, S. (1999). Release of methyl methacrylate from heat-cured and autopolymerized resins: cytotoxicity testing related to residual monomer. Australian Dental Journal, Vol.44, 25-30, ISSN 00450421

Keenan, P.L.J.; Radford, D.R. \& Clark, R.K.F. (2003). Dimensional change in complete dentures fabricated by injection molding and microwave processing. Journal of Prosthetic Dentistry, Vol.89, 37-44, ISSN 0022-3913

Keller, J.C. \& Lautenschlager, E.P. (1985). Porosity reduction and its associated effect on the diametral tensile strength of activated acrylic resins. Journal of Prosthetic Dentistry, Vol.53, 374-379, ISSN 0022-3913

Kimura, H.; Teraoka, F.; Ohnishi, H.; Saito, T. \& Yato, M. (1983). Applications of microwave for dental technique, Part 1: Dough-forming and curing of acrylic resins. The Journal of Osaka University Dental School, Vol.23, 43-49, ISSN 0473-4599

Kimura, H.; Teraoka, F. \& Saito, T. (1984). Applications of microwave for dental technique (Part 2): adaptability of cured acrylic resins. The Journal of Osaka University Dental School, Vol.23, 43-49, ISSN 0473-4599

Kimura, H.; Teraoka, F. \& Sugita, M. (1987). Application of microwave for dental technique, Part 3: Development of model materials for microwave polymerization. The Journal of Osaka University Dental School, Vol.27, 41-50, ISSN 0473-4599

Lai, C.P.; Tsai, M.H.; Chen, M.; Chang, H.S. \& Tay, H.H. (2004). Morphology and properties of denture acrylic resins cured by microwave energy and conventional water bath. Dental Materials, Vol.20, No.2, (Feb) 133-141, ISSN 0109-5641

Lamb, D.J.; Ellis, B. \& Priestley, D. (1983). The effects of process variables on levels of residual monomer in autopolymerizing dental acrylic resin. Journal of Dentistry, Vol.11, 80-88, ISSN 0300-5712

Lbay, S.G.; Gu Vener, S. \& Alkumru, H.N. (1994). Processing dentures using a microwave technique. Journal of Oral Rehabilitation, Vol.21, 103-109, ISSN 0305-182X

Levin, B.; Sanders, J.L. \& Reitz, P.V. (1989). The use of microwave for processing acrylic resins. Journal of Prosthetic Dentistry, Vol.61, 381-383, ISSN 0022-3913

Lin, J.J.; Cameron, S.M.; Runyan, D.A. \& Craft, D.W. (1999). Disinfection of denture base acrylic resin. Journal of Prosthetic Dentistry, Vol.81, No.2, (Feb) 202-206, ISSN 00223913

Machado, A.L.; Breeding, L.C. \& Puckett, A.D. (2005). Effect of microwave disinfection on the hardness and adhesion of two resilient liners. Journal of Prosthetic Dentistry, Vol.94, 183-189, ISSN 0022-3913

Machado, A.L.; Breeding, L.C. \& Puckett, A.D. (2006). Effect of microwave disinfection procedures on torsional bondstrengths of two hard chairside denture reline materials. Journal of Prosthodontics, Vol.15, 337-344, ISSN 1059-941X

Machado, A.L.; Breeding, L.C.; Vergani, C.E. \& Perez, L.E.C. (2009). Hardness and surface roughness of reline and denture base acrylic resins after repeated disinfection procedures. Journal of Prosthetic Dentistry, Vol.102, 115-122, ISSN 0022-3913

Machado, C.; Rizzatti-Barbosa, C.M.; Gabriotti, M.; Joia, F.; Ribeiro, M.C. \& Sous, R.L.S. (2004). Influence of mechanical and chemical polishing in the solubility of acrylic 
resins polymerized by microwave irradiation and conventional water bath. Dental Materials, Vol.20, 565-569, ISSN 0109-5641

May, K.B.; Razzoog, M.E.; Koran, A. 3rd \& Robinson, E. (1992). Denture base resins: comparison study of color stability. Journal of Prosthetic Dentistry, Vol.68, No.1, (Jul) 78-82, ISSN 0022-3913

May, K.B.; Shotwell, J.R.; Koran, A. \& Wang, R.F. (1996). Color stability: denture base resins processed with the microwave method. Journal of Prosthetic Dentistry, Vol.76, 581589, ISSN 0022-3913

McCabe, J.F.\& Basker, R.M. (1976) Tissue sensitivity to acrylic resin. British Dental Journal, Vol.140, No.10, (May) 347-350, ISSN 0007-0610

Meloto, C.B.; Silva-Concílio, L.R.; Machado, C.; Ribeiro, M.C.; Joia, F.A. \& Rizzatti-Barbosa, C.M. (2006). Water Sorption of Heat-Polymerized Acrylic Resins Processed in Mono and Bimaxillary Flasks. Brazilian Dental Journal, Vol.17, No.2, 122-125, ISSN 01036440

Memon, M.S.; Yunus, N. \& Razak, A.A. (2001). Some mechanical properties of a highly cross-linked, microwave-polymerised, injection molded denture base polymer. The International Journal of Prosthodontics, Vol.14, 214-218, ISSN 0893-2174

Mima, E.G.O; Pavarina, A.C.; Neppelenbroek, K.H.; Vergani, C.E.; Spolidorio, D.M. \& Machado, A.L. (2008). Effect of different exposure times on microwave irradiation on the disinfection of a hard chairside reline resin. Journal of Prosthodontics, Vol.17, No.4, (Jun) 312-317, ISSN 1059-941X

Montagner, H.; Montagner, F.; Braun, K.O.; Peres, P.E. \& Gomes, B.P. (2009). In vitro antifungal action of different substances over microwaved-cured acrylic resins. Journal of Applied Oral Science, Vol.17, 432-435, ISSN 1678-7757

Moura, J.; Silva, W.J.; Pereira, T.; Del Bel Cury, A. \& Rodrigues Garcia, R.C.M. (2006). Influence of acrylic resin polymerization methods and saliva on the adherence of four Candida species. Journal of Prosthetic Dentistry, Vol.96, 205-211, ISSN 0022-3913

Najdovski, L.; Dragas, A.Z. \& Kotnik, V. (1991). The killing activity of microwaves on some nonsporogenic and sporogenic medically important bacterial strains. The Journal of Hospital Infection, Vol.19, 239-247, ISSN 0195-6701

Negreiros, W.A.; Consani, R.L.X.; Verde, M.A.R.L.; Silva, A.M. \& Pinto, L.P. (2009). The role of polymerization cycle and post-pressing time on tooth movement in complete dentures. Brazilian Oral Research, Vol.23, No.4, (Oct-Dec) 467-472, ISSN 1806-8324

Nelson, M.W.; Kotwal, K.R. \& Sevedge, S.R. (1991). Changes in vertical dimension of occlusion in conventional and microwave processing of complete dentures. Journal of Prosthetic Dentistry, Vol.65, 306-308, ISSN 0022-3913

Neppelenbroek, K.H.; Pavarina, A.C.; Spolidorio, D.M.; Vergani, C.E.; Mima, E.G. \& Machado, A.L. (2003). Effectiveness of microwave sterilization on three hard chairside reline resins. The International Journal of Prosthodontics, Vol.16, 616-620, ISSN 0893-2174

Nikawa, H.; Jin, C.; Hamada, T.; Makihira, S.; Kumagai, H. \& Murata, H. (2000). Interactions between thermal cycled resilient denture lining materials, salivary and serum pellicles and Candida albicans in vitro. Part II. Effects on fungal colonization. Journal of Oral Rehabilitation, Vol.27, 124-130, ISSN 0305-182X 
Nishii, M. (1968). Studies on the curing of denture base resins with microwave irradiation: with particular reference to heat-curing resins. The Journal of Osaka University Dental School, Vol.2, 23-40, ISSN 0473-4599

Novais, P.M.M.R.; Giampaolo, E.T.; Vergani, C.E.; Machado, A.L.; Pavarina, A.C. \& Jorge, J.H. (2009). The occurrence of porosity in reline acrylic resins. Effect of microwave disinfection. Gerodontology, Vol.26, 65-71, ISSN 0734-0664

Oliveira, V.M.; Leon, B.L.; Del Bel Cury, A.A. \& Consani, S. (2003). Influence of number and position of flasks in the monomer release, Knoop hardness and porosity of a microwave-cured acrylic resin. Journal of Oral Rehabilitation, Vol.30, 1104-1108, ISSN 0305-182X

Paranhos, H.F.O.; Davi, L.R.; Peracini, A.; Soares, R.B.; Lovato, C.H.S. \& Souza, R.F. (2009). Comparison of Physical and Mechanical Properties of Microwave-Polymerized Acrylic Resin after Disinfection in Sodium Hypochlorite Solutions. Brazilian Dental Journal, Vol.20, No.4, 331-335, ISSN 0103-6440

Pavan, S.; Arioli Filho, J.N.; Dos Santos, P.H. \& Mollo, F.A. Jr (2005). Effect of microwave treatments on dimensional accuracy of maxillary acrylic resin denture base. Brazilian Dental Journal, Vol.16, 119- 123, ISSN 0103-6440

Pavan, S.; Arioli Filho, J.N.; Santos, P.H.; Nogueira, S.S. \& Batista, A.U.D. (2007). Effect of Disinfection Treatments on the Hardness of Soft Denture Liner Materials. Journal of Prosthodontics, Vol.20, 101-106, ISSN 1059-941X

Pavarina, A.C.; Neppelenbroek, K.H.; Guinesi, A.S.; Vergani, C.E.; Machado, A.L. \& Giampaolo, E.T. (2005). Effect of microwave disinfection on the flexural strength of hard chairside reline resins. Journal of Dentistry, Vol.33, 741-748, ISSN 0300-5712

Pavarina, A.C.; Pizzolitto, A.C.; Machado, A.L.; Vergani, C.E. \& Giampaolo, E.T. (2003). An infection control protocol: effectiveness of immersion solutions to reduce the microbial growth on dental prostheses. Journal of Oral Rehabilitation, Vol.30, 532-536, ISSN 0305-182X

Pero, A.C.; Barbosa, D.B.; Marra, J.; Ruvolo-Filho, A.C. \& Compagnoni, M.A. (2007). Influence of Microwave Polymerization Method and Thickness on Porosity of Acrylic Resin. Journal of Prosthodontics, Vol.17, No.2, 125-129, ISSN 1059-941X

Peyton, F.A. (1950). Packing and processing denture base resins. Journal of the American Dental Association, Vol.40, 521-526, ISSN 0002-8177

Phoenix, R.D.; Mansueto, M.A.; Ackerman, N.A. \& Jones, R.E. (2004). Evaluation of mechanical and thermal properties of commonly used denture base resins. Journal of Prosthodontics, Vol.13, 17-27, ISSN 1059-941X

Polyzois, G.L.; Karkazis, H.C.; Zissis, A.J. \& Demetriou, P.P. (1987). Dimensional stability of denture processed in boilable acrylic resins: a comparative study. Journal of Prosthetic Dentistry, Vol.57, No.5, 639-647, ISSN 0022-3913

Polyzois, G.L.; Tarantili, P.A.; Frangou, M.J. \& Andreopoulos, A.G. (2001). Fracture force, deflection at fracture, and toughness of repaired denture resin subjected to microwave polymerization or reinforced with wire or glass fiber. Journal of Prosthetic Dentistry, Vol.86, No.6, (Dec) 613-619, ISSN 0022-3913

Polyzois, G.L.; Yannikakis, S.A. \& Zissis, A.J. (1999). Color stability of visible light-cured, hard direct denture reliners: an in vitro investigation. The International Journal of Prosthodontics, Vol.12, 140-146, ISSN 0893-2174 
Polyzois, G.L.; Zissis, A.J. \& Yannikakis, S.A. (1995). The effect of glutaraldehyde and microwave disinfection on some properties of acrylic denture resin. The International Journal of Prosthodontics, Vol.8, 150-154, ISSN 0893-2174

Polyzois, G.L., Handley, R.W. \& Stafford, G.D. (1995). Repair strength of denture base resins using various methods. European Journal of Prosthodontics and Restorative Dentistry, Vol.3, No.4, (Jun) 183-186, ISSN 0965-7452

Rached, R.N. \& Del Bel Cury, A.A. (2001). Heat-cured acrylic resin repaired with microwave-cured one: Bond strength and surface texture. Journal of Oral Rehabilitation, Vol.28, 370-375, ISSN 0305-182X

Reitz, P.V.; Sanders, J.L. \& Levin, B. (1985). The curing of denture acrylic resins by microwave energy. Physical properties. Quintessence International, Vol.16, 547-551, ISSN 0033-6572

Ribeiro, D.G.; Pavarina, A.C.; Machado, A.L.; Giampaolo, E.T. \& Vergani, C.E. (2008). Flexural strength and hardness of reline and denture base acrylic resins after different exposure times of microwave disinfection. Quintessence International, Vol.39, No.10, (Nov) 833-840, ISSN 0305-182X

Rizzatti-Barbosa CM, Del Bel Cury AA \& Panzeri H. (1995). Influence of water sorption and microwave polymerization in the in adaptability of dentures. Revista deOdontologia da Universidade Sao Paulo Vol.9, 197-206, ISSN 0103-0663

Rizzatti-Barbosa, C.M.; Machado, C.; Jóia, F.A. \& Sousa, R.L.S. (2005). A method to reduce tooth movement of complete dentures during microwave irradiation processing. Journal of Prosthetic Dentistry, Vol.94, No.3, 301-302, ISSN 0022-3913

Rizzatti-Barbosa, C.M. \& Ribeiro da Silva, M.C. (2009). Influence of Double Flask Investing and Microwave Heating on the Superficial Porosity, Surface Roughness, and Knoop Hardness of Acrylic Resin. Journal of Prosthodontics, Vol.18, 503-506, ISSN 1059-941X

Rodrigues Garcia, R.C.M; Souza Jr, J.A.; Rached, R.N. \& Del Bel Cury, A.A. (2004). Effect of denture cleansers on the surface roughness and hardness of a microwave-cured acrylic resin and dental alloys. Journal of Prosthodontics, Vol.13, 173-178, ISSN 1059941X

Rodrigues Garcia, R.C.M. \& Del Bel Cury, A.A. (1996). Accuracy and porosity of denture bases submitted to two polymerization cycles. Indian Journal of Dental Research, Vol.7, 122-126, ISSN 0970-9290

Rohler, D.M. \& Bulard, R.A. (1985). Microwave sterilization. Journal of the American Dental Association, Vol.110, 194-198, ISSN 0002-8177

Sadamori, S.; Ganefiyanti, T.; Hamada, T. \& Arima, T. (1994). Influence of thickness and location on the residual monomer content of denture base cured by three processing methods. Journal of Prosthetic Dentistry, Vol.72, No.1, (Jul) 19-22, ISSN 0022-3913

Sadamori, S., Ishii,T. \& Hamada, T. (1997). Influence of thickness on the linear dimensional change, warpage, and water uptake of a denture base resin. The International Journal of Prosthodontics, Vol.10, No.1, 35-43, ISSN 0893-2174

Salim, S.; Sadamori, S. \& Hamada, T. (1992). The dimensional accuracy of rectangular acrylic resin specimens cured by three denture base processing methods. Journal of Prosthetic Dentistry, Vol.67, 879-881, ISSN 0022-3913 
Sanders, J.L.; Levin, B. \& Reitz, P.V. (1991). Comparison of the adaptation of acrylic resin cured by microwave energy and conventional water bath. Quintessence International, Vol.22, 181-186, ISSN 0305-182X

Sanders, J.L.; Levin, B. \& Reitz, P.V.(1987). Porosity in denture acrylic resins cured by microwave energy. Quintessence International, Vol.18, 453-456, ISSN 0305-182X

Sanita, P.V.; Vergani, C.E.; Giampaolo, E.T.; Pavarina, A.C. \& Machado, A.L. (2009). Growth of Candida species on complete dentures: effect of microwave disinfection. Mycoses, Vol.52, No.2, (Mar) 154-160, ISSN 0933-7407

Sarac, Y.S.; Sarac, D.; Kulunk, T. \& Kulunk, S. (2005). The effect of chemical surface treatments of different denture base resins on the shear bond strength of denture repair. Journal of Prosthetic Dentistry, Vol.94, 259-266, ISSN 0022-3913

Sartori, E.A.; Schmidt, C.B.; Mota, E.G.; Hirakata, L.M. \& Shinkai, R.S. (2008). Cumulative effect of disinfection procedures on the microhardness and tridimensional stability of a poly(methyl methacrylate) denture base resin. Journal of Biomedical Materials Research. Part B, Applied Biomaterials, Vol.86B, 360-364, ISSN 1552-4973

Sartori, E.A.; Schmidt, C.B.; Walber, L.F. \& Shinkai, R.S. (2006). Effect of microwave disinfection on denture base adaptation and resin surface roughness. Brazilian Dental Journal, Vol.17, 195-200, ISSN 0103-6440

Schibayama, R.; Gennari Filho, H.; Mazaro, J.V.Q.; Vedovatto, E. \& Assunção, W.G. (2009). Effect of flasking and polymerization techniques on tooth movement in complete denture processing. Journal of Prosthodontics, Vol.18, No.3, (Apr) 259-264, ISSN 1059-941X

Schneider, R.L.; Curtis, E.R.; Clancy, J.M.S. (2002). Tensile bond strength of acrylic resin denture teeth to a microwave- or heat-processed denture base. Journal of Prosthetic Dentistry, Vol.88, 145-150, ISSN 0022-3913

Seo, R.S.; Vergani, C.E.; Pavarina, A.C.; Compagnoni, M.A. \& Machado, A.L. (2007). Influence of microwave disinfection on the dimensional stability of intact and relined acrylic resin denture bases. Journal of Prosthetic Dentistry, Vol.98, No.3, (Sept) 216-223, ISSN 0022-3913

Setlow, P. (2006). Spores of Bacillus subtilis: their resistance to and killing by radiation, heat and chemicals. Journal of Applied Microbiology, Vol.101, No.3, (Sept) 514-525, ISSN 1364-5072

Shlosberg, S.R.; Goodacre, C.J.; Munoz, C.A.; Moore, B.K. \& Schnell, R.J. (1989). Microwave energy polymerization of poly(methyl methacrylate) denture base resin. The International Journal of Prosthodontics, Vol.2, 453-458, ISSN 0893-2174

Silva, M.M.; Vergani, C.E.; Giampaolo, E.T.; Neppelenbroek, K.H.; Spolidorio, D.M. \& Machado, A.L. (2006). Effectiveness of microwave irradiation on the disinfection of complete dentures. The International Journal of Prosthodontics, Vol.19, 288-293, ISSN 0893-2174

Smith, L.T.; Powers, J.M. \& Ladd, D. (1992). Mechanical properties of new denture resins polymerized by visible light, heat, and microwave energy. The International Journal of Prosthodontics, Vol.5, 315-320, ISSN 0893-2174

Souza Jr, J.A.; Rodrigues-Garcia, R.C.M.; Moura, J.S. \& Del Bel Cury, A.A. (2006). Knoop hardness of visible light-polymerized acrylic resins. Journal of Applied Oral Science, Vol.14, No.3, 208-212, ISSN 1678-7757 
Stafford, G.D. \& Handley, R.W. (1975). Transverse bend testing of denture base polymers. Journal of Dentistry, Vol.3, No.6, 251-255, ISSN 0300-5712

Stafford, G.D. \& Huggett, R. (1978). Creep and hardness testing of some denture base polymers. Journal of Prosthetic Dentistry, Vol.39, No.6, 682-687, ISSN 0022-3913

Sweeney, W.T.; Paffenbarger, G.C. \& Beall, J.R. (1942). Acrylic resins for dentures. Journal of the American Dental Association, Vol.29, 7-33, ISSN 0002-8177

Tacir, I.H.; Kama, J.D.; Zortuk, M. \& Eskimez, S. (2006). Flexural properties of glass fibre reinforced acrylic resin polymers. Australian Dental Journal, Vol.51, No.1, 52-56, ISSN 0045-0421

Tager, A. (1978). Physical Chemistry of Polymers, pp.593-627, Mir Publisher, Moscow

Takahashi, Y.; Chai, J. \& Kawaguchi, M. (1998). Effect of water sorption on the resistance to plastic deformation of a denture base material relined with four different denture reline materials. The International Journal of Prosthodontics, Vol.11, 49-54, ISSN 08932174

Takamata, T.; Setcos, J.C.; Phillips, R.W. \& Boone, M.E. (1989). Adaptation of acrylic resin dentures as influenced by the activation mode of polymerization. Journal of the American Dental Association, Vol.119, 271-276, ISSN 0002-8177

Taylor, P.B. (1941). Acrylic resins: their manipulation. Journal of the American Dental Association, Vol.28, 373-387, ISSN 0002-8177

Thomas, C.J. \& Webb, B.C. (1995). Microwaving of acrylic resin dentures. The European Journal of Prosthodontics and Restorative Dentistry, Vol.3, No.4, 179-182, ISSN 09657452

Truong, V.T. \& Thomaz, F.G. (1988). Comparison of denture acrylic resins cured by boiling water and microwave energy. Australian Dental Journal, Vol.33, 201-204, ISSN 00450421

Uchida, K., Okamoto, F., Ogata, K. \& Sato, T. (1989). Dimensional accuracy of microwavecured denture base resin. Nippon Hotetsu Shika Gakkai Zasshi, Vol.33, No.1, 114-118, ISSN 0389-5386

Urabe, H.; Nomura, Y.; Shirai, K.; Yoshioka, M. \& Shintani, H. (1999). Influence of polymerization initiator for base monomer on microwave curing of composite resin inlays. Journal of Oral Rehabilitation, Vol.26, 442-446, ISSN 0305-182X

Urban, V.M.; Machado, A.L.; Oliveira, R.V.; Vergani, C.E.; Pavarina, A.C. \& Cass, Q.B. (2007). Residual monomer of reline acrylic resins. Effect of water-bath and microwave postpolymerization treatments. Dental Materials, Vol.23, 363-368, ISSN 0109-5641

Vergani, C.E.; Seo, R.S.; Pavarina, A.C. \& Reis, J.M.S.N. (2005). Flexural strength of autopolymerizing denture reline resins with microwave postpolymerization treatment. Journal of Prosthetic Dentistry, Vol.93, 577-583, ISSN 0022-3913

Wallace, P.W.; Graser, G.N.; Myers, M.L. \& Proskin, H.M. (1991). Dimensional accuracy of denture resin cured by microwave energy. Journal of Prosthetic Dentistry, Vol.66, 403-408, ISSN 0022-3913

Webb, B.C.; Thomas, C.J.; Harty, D.W. \& Willcox, M.D. (1998). Effectiveness of two methods of denture sterilization. Journal of Oral Rehabilitation, Vol.25, 416-423, ISSN 0305$182 X$

Witt S, H.P. (1990). Cross-infection hazards associated with the use of pumice in dental laboratories. Journal of Dentistry, Vol.18, 281-283, ISSN 0300-5712 
Wolfaardt, J.F.; Cleaton-Jones, P. \& Fatti, P. (1986). The occurrence of porosity in a heatcured poly(methyl methacrylate) denture base resin. Journal of Prosthetic Dentistry, Vol.55, 393-400, ISSN 0022-3913

Wong, D.M.S.; Cheng, L.Y.Y.; Chow, T.W. \& Clark, R.K.F. (1999). Effect of processing method on the dimensional accuracy and water sorption of acrylic resin dentures. Journal of Prosthetic Dentistry, Vol.81, 300-304, ISSN 0022-3913

Yagi T, Marimoto T, Hidako O, Iwata K, Masuda Y, Kabayashi M, Takada K. Adjustment of the oclusal vertical dimension in bit raised guinea pig. Journal of Dental Research, Vol.82,123-130, ISSN 0022-0345

Yannikakis, S.; Zissis, A.; Polyzois, G. \& Andreopoulos, A. (2002). Evaluation of porosity in microwave-processed acrylic resin using a photographic method. Journal of Prosthetic Dentistry, Vol.87, 613-619, ISSN 0022-3913

Yeo, C.B.; Watson, I.A.; Stewart-Tull, D.E. \& Koh, V.H. (1999). Heat transfer analysis of Staphylococcus aureus on stainless steel with microwave radiation. Journal of Applied Microbiology, Vol.87, No.3, 396-401, ISSN 1364-5072

Yunus, N.; Rashid, A.A.; Azmi, L.L. \& Abu-Hassan, M.I. (2005). Some flexural properties of a nylon denture base polymer. Journal of Oral Rehabilitation, Vol.32, 65-71, ISSN 0305-182X

Yunus, N.; Harrison, A. \& Hugget, R. (1994). Effect of microwave irradiation on the flexural strength and residual monomer levels of an acrylic resin repair material. Journal of Oral Rehabilitation, Vol.21, 641-648. ISSN 0305-182X

Zissis, A.J.; Polyzois, G.L.; Yannikakis, S.A. \& Harrison, A. (2000). Roughness of denture materials: a comparative study. The International Journal of Prosthodontics, Vol.13, 136-140, ISSN 0893-2174 


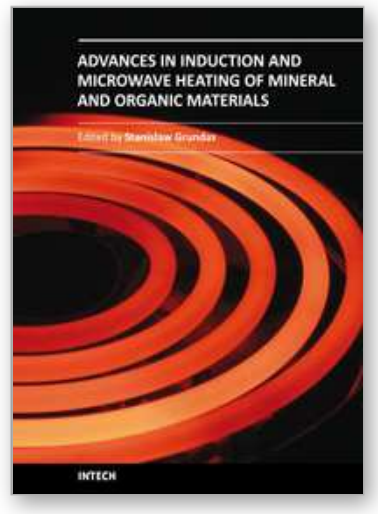

\author{
Advances in Induction and Microwave Heating of Mineral and \\ Organic Materials
}

Edited by Prof. StanisÅ,aw Grundas

ISBN 978-953-307-522-8

Hard cover, 752 pages

Publisher InTech

Published online 14, February, 2011

Published in print edition February, 2011

The book offers comprehensive coverage of the broad range of scientific knowledge in the fields of advances in induction and microwave heating of mineral and organic materials. Beginning with industry application in many areas of practical application to mineral materials and ending with raw materials of agriculture origin the authors, specialists in different scientific area, present their results in the two sections: Section 1-Induction and Microwave Heating of Mineral Materials, and Section 2-Microwave Heating of Organic Materials.

\title{
How to reference
}

In order to correctly reference this scholarly work, feel free to copy and paste the following:

Célia M. Rizzatti-Barbosa, Altair A. Del Bel Cury and Renata C. M. Rodrigues Garcia (2011). The Use of Microwave Energy in Dental Prosthesis, Advances in Induction and Microwave Heating of Mineral and Organic Materials, Prof. StanisÅ,aw Grundas (Ed.), ISBN: 978-953-307-522-8, InTech, Available from:

http://www.intechopen.com/books/advances-in-induction-and-microwave-heating-of-mineral-and-organicmaterials/the-use-of-microwave-energy-in-dental-prosthesis

\section{INTECH}

open science | open minds

\section{InTech Europe}

University Campus STeP Ri

Slavka Krautzeka 83/A

51000 Rijeka, Croatia

Phone: +385 (51) 770447

Fax: +385 (51) 686166

www.intechopen.com

\section{InTech China}

Unit 405, Office Block, Hotel Equatorial Shanghai

No.65, Yan An Road (West), Shanghai, 200040, China

中国上海市延安西路65号上海国际贵都大饭店办公楼 405 单元

Phone: +86-21-62489820

Fax: +86-21-62489821 
(C) 2011 The Author(s). Licensee IntechOpen. This chapter is distributed under the terms of the Creative Commons Attribution-NonCommercialShareAlike-3.0 License, which permits use, distribution and reproduction for non-commercial purposes, provided the original is properly cited and derivative works building on this content are distributed under the same license. 